\title{
Decoherence in quantum walks - a review
}

\author{
VIV KENDON ${ }^{\dagger}$ \\ School of Physics and Astronomy, University of Leeds, Leeds, LS2 9JT, U.K. \\ Email: v.kendon@leeds.ac.uk
}

Received 27 November 2006

The development of quantum walks in the context of quantum computation, as generalisations of random walk techniques, has led rapidly to several new quantum algorithms. These all follow a unitary quantum evolution, apart from the final measurement. Since logical qubits in a quantum computer must be protected from decoherence by error correction, there is no need to consider decoherence at the level of algorithms. Nonetheless, enlarging the range of quantum dynamics to include non-unitary evolution provides a wider range of possibilities for tuning the properties of quantum walks. For example, small amounts of decoherence in a quantum walk on the line can produce more uniform spreading (a top-hat distribution), without losing the quantum speed up. This paper reviews the work on decoherence, and more generally on non-unitary evolution, in quantum walks and suggests what future questions might prove interesting to pursue in this area.

\section{Contents}

1 Introduction

2 Coined (discrete-time) quantum walks

2.1 Coined quantum walk on an infinite line

2.2 Coined quantum walk on an $N$-cycle: mixing times

2.3 Periodicity in coined quantum walks on cycles

2.4 Coined quantum walks on higher dimensional graphs

2.5 Coined quantum walks on regular lattices

2.6 Coined quantum walk on the hypercube: hitting times 1180

3 Continuous-time quantum walks

3.1 Continuous-time quantum walks on general graphs 1180

3.2 Continuous-time quantum walk on the line 1182

3.3 Continuous-time quantum walk on an $N$-cycle and other circulant graphs 1183

3.4 Continuous-time quantum walk on the hypercube 1184

4 Algorithms using quantum walks 1185

4.1 Quantum walk searching 1185

4.2 'Glued trees' algorithm 1186

5 Decoherence in coined quantum walks $\quad 1187$

5.1 Effects in the walk on the line 1189

5.2 Dephasing the coin in the walk on the line 1192

$\dagger$ The author is funded by a Royal Society University Research Fellowship. 
5.3 Decohering the position in the walk on the line

1193

5.4 Multiple coins in the walk on the line

5.5 Effects in the walk on the $N$-cycle

5.6 Effects in the walk on the hypercube 1201

6 Decoherence in continuous-time walks 1203

6.1 Effects in the walk on the line 1204

6.2 Effects in the walk on the $N$-cycle 1205

6.3 Effects in the walk on the hypercube 1210

7 Quantum walks in physical systems 1212

8 Quantum walks on directed graphs 1214

9 Summary and outlook 1215

References 1216

\section{Introduction}

The study of quantum versions of random walks is easily motivated. They have provided the engine for several new quantum algorithms, they are of mathematical interest in their own right, and in physical systems they form a simple example of coherent quantum control over single atoms or photons. They have also found applications from the early days of quantum computation as the clock mechanism in a Feynman computer (Feynman 1986). The role of decoherence requires more explanation. In physical systems we must, of course, consider the effects of decoherence when designing our experiments, but this alone does not qualify it as a subject for more general study. Decoherence plays a fundamental role in moving from the quantum to the classical regime. Quantum walks are simple systems in which the intricacies of decoherence can be studied both analytically and numerically, making connections with fields such as quantum chaos. Taking a broad view of decoherence as any process that tends to reduce quantum coherence, we find that it provides a method for tuning quantum random walks to improve their algorithmic properties. Instead of restricting ourselves to pure quantum dynamics, we can include non-unitary operations in our quantum random walk algorithms, thereby enlarging the toolbox for controlling their behaviour.

The origins of quantum walks can be traced back to the dynamics of quantum diffusion, which has been well studied in the physics literature, see for example, Feynman et al. (1964), mostly as models for physical particles moving on regular lattices. Studies of quantum dynamics using a discrete time step, described as 'quantum random walks', appeared from the late 1980s, including Gudder (1988), Grossing and Zeilinger (1988) and Aharonov et al. (1992), the last with quantum optical applications in mind. Meyer (1996b) studied similar systems with the aim of defining quantum cellular automata (Meyer 1996a). The current surge of interest in the context of quantum information started with Farhi and Gutmann (1998) for continuous-time quantum walks, and continued with Aharonov et al. (2001), Ambainis et al. (2001) and Nayak and Vishwanath (2000) for discrete-time quantum walks, all with the goal of applying them to quantum algorithms. As with classical random walks, there are a wide range of possible dynamics that fit within 
the general concept: for example, Gottlieb (2004) and Gottlieb et al. (2005) defined a discrete-time walk with a continuous space and a coin with a continuous set of states.

In this review we will start with a short overview of purely quantum versions of random walks as studied by the quantum information community in their quest for new quantum algorithms, then in later sections we will consider the added effects of decoherence. We will cover discrete-time, coined quantum walks in Section 2, then continuous-time quantum walks in Section 3. We mention algorithmic applications in Section 4, briefly describing two well-known algorithms using quantum walks. In Sections 5 and 6 we consider the effects of decoherence in quantum walks, focusing mainly on its use as a tool for enhancing computational speed up. There are several proposals for implementing a quantum walk directly in a physical system, and most of these studies also contribute an analysis of the decoherence relevant to the physical system in question: we provide a short summary of these studies in Section 7. In general, we deal only with quantum walks on undirected graphs, with a brief summary of what is known about the largely unexplored realm of directed graphs in Section 8.

This review will assume a basic familiarity with quantum mechanics and quantum information theory. Those wishing to get up to speed on these areas in the context of quantum walks are referred to the excellent and comprehensive introductory review in Kempe (2003a), which just assumes a basic knowledge of quantum mechanics. Those desiring a gentle overview of quantum walks in the context of quantum computing should see Kendon (2006b). A brief but accessible review of quantum walk algorithms from a computer science perspective (with no mention of decoherence) may be found in Ambainis (2003).

The mathematical results and proofs for quantum walks without decoherence have been presented in great detail and elegance in the original literature: this review summarises what is known without presenting formal proofs but with ample references for those interested in pursuing them further. Fewer analytic results have been derived for decoherence effects: those that exist are presented in more detail, along with summaries of many related ideas, highlighting the myriad of open avenues that remain to be explored.

\section{Coined (discrete-time) quantum walks}

In this section we describe quantum walks taking place in a discrete space of positions, with an evolution using discrete time steps. Just as there are many ways to express the dynamics of classical random walks, and many variations on the basic 'drunkard's walk', the plethora of different studies of quantum walks have proliferated an equally varied set of notations. For this review, we have chosen one of the more commonly used approaches, and try to maintain a consistent notation, with mention of alternative methods where appropriate. To keep the notation simple, we have taken the liberty of using the same symbols for common quantities throughout, with the precise definition implied by the context. For example, $P(x, t)$ is a probability distribution over a set of positions denoted by $x$ at a time $t$ during the evolution. In the section on the quantum walk on the line, $P(x, t)$ is for the walk on the line, in the section for the walk on a cycle $P(x, t)$ is for the 
walk on a cycle, and so on. Additional independent variables may appear where needed: for example, $P(x, a, t)$ is a probability distribution with the coin state $a$ also specified.

We begin with a brief description of one of the simplest examples, the quantum walk on the line. This will serve both to set up our notation and to indicate the methods of solution. It will also illustrate the first key difference between quantum and classical random walks, the enhanced rate of spreading. This is followed by a discussion of quantum walks on cycles, illustrating several more key differences between classical and quantum random walks. We will conclude this section with a brief review of coined quantum walks in higher dimensions, on general graphs and regular lattices.

\subsection{Coined quantum walk on an infinite line}

In much the same way as we now know almost everything about the properties and possible states of two qubits - though quantum computers will clearly need far more than two qubits to be useful - the simple quantum walk on a line has now been thoroughly studied (see, for example, Ambainis et al. (2001), Bach et al. (2004), Yamasaki et al. (2002), Kendon and Tregenna (2003), Brun et al. (2003a; 2003c), Konno et al. (2004), Konno (2002) and Carteret et al. (2003)), though there is no suggestion that it will lead to useful quantum walk algorithms by itself.

First, recall the classical random walk dynamics for which we are seeking a quantum counterpart. The walker starts at the origin of an infinite line of discrete points (labelled $\ldots-2,-1,0,1,2 \ldots)$ and tosses an unbiased, two-sided coin. If the coin lands 'heads' the walker makes one step in the positive direction; if it lands 'tails' the walker steps in the negative direction. This process is repeated $T$ times, and the position of the walker is noted: $-T \leqslant x \leqslant T$. If the random walk is repeated many times, the probability distribution $P(x, T)$ obtained is binomial, as is well known and easily shown. The standard deviation of $P(x, T)$ is $\sqrt{T}$, that is, the walker is found on average $\sqrt{T}$ steps from the origin after $T$ steps of the random walk.

One obvious approach to creating a quantum counterpart of a classical random walk is to have the quantum walker follow all possible classical random walks in superposition. This is not feasible in the discrete-time quantum walk, as was shown in Meyer (1996b), because it is not reversible, and therefore not unitary (which all pure quantum dynamics is required to be). We thus take a different approach to making the quantum walk dynamics as similar as possible to the classical random walk by using a quantum coin. Historically, the role of the quantum coin has caused much confusion, and an equivalent formulation presented in Watrous (2001) using directed graphs seems to be preferred by some computer scientists. For brevity and clarity, in this review we will stick to the formulation using a quantum coin, with brief mention of the Watrous variant in Section 8. The quantum walker on the line starts at the origin and tosses a two-state quantum system (a qubit). The qubit coin - also called 'chirality' with the two states labelled 'left' and 'right' in many papers - can be in a superposition of both states, say

$$
|\psi(t=0)\rangle=\alpha|0,-1\rangle+\beta e^{i \phi}|0,+1\rangle,
$$

where the 'ket' $|\cdot\rangle$ is a standard quantum notation to indicate the complex vector of a 
pure quantum state. On the right-hand side the kets are basis states: the first entry is the position on the line ( $x=0$ in this case), and the second is the state of the quantum coin, where we choose to label the two states $| \pm 1\rangle$. There is an arbitrary phase $0 \leqslant \phi<2 \pi$, while $\alpha$ and $\beta$ are real numbers, with normalisation $\alpha^{2}+\beta^{2}=1$. The quantum walker then steps in both directions according to the state of the quantum coin, giving

$$
|\psi(t=1)\rangle=\alpha|-1,-1\rangle+\beta e^{i \phi}|1,+1\rangle .
$$

Keeping to a pure quantum (that is, unitary) evolution for the moment, the coin toss and step can be written as unitary operators $\mathbf{C}$ and $\mathbf{S}$. A single step of the quantum random walk is thus

$$
|\psi(t+1)\rangle=\mathbf{S C}|\psi(t)\rangle
$$

where $|\psi(t)\rangle$ can be expanded as a superposition of basis states,

$$
|\psi(t)\rangle=\sum_{x, c} a_{x, c}(t)|x, c\rangle
$$

with complex coefficients $a_{x, c}(t)$ satisfying $\sum_{x, c}\left|a_{x, c}(t)\right|^{2}=1$. After $T$ steps we have

$$
|\psi(T)\rangle=(\mathbf{S C})^{T}|\psi(0)\rangle .
$$

The shift operator $\mathbf{S}$ has already been specified implicitly by equations (1) and (2): it can be defined by its operation on a basis state $|x, c\rangle$,

$$
\mathbf{S}|x, c\rangle=|x+c, c\rangle .
$$

We still need to specify the coin toss $\mathbf{C}$, which can in principle be any unitary operator on the space of a qubit. The equivalence up to a bias (probability $\eta, 0 \leqslant \eta \leqslant 1$, to move in the positive direction, $(1-\eta)$ in the negative direction) of all coin operators in the walk on a line has been noted by several authors (Ambainis et al. 2001; Bach et al. 2004; Yamasaki et al. 2002). Expressing the unitary operator as a matrix we have

$$
\mathbf{C}_{2}=\left(\begin{array}{rr}
\sqrt{\eta} & \sqrt{1-\eta} \\
\sqrt{1-\eta} & -\sqrt{\eta}
\end{array}\right),
$$

which is thus the only possible type of coin for the quantum walk on a line. For $\eta=1 / 2$ (unbiased), $\mathbf{C}_{2}^{(\mathrm{H})}$ is commonly known as a Hadamard operator,

$$
\mathbf{C}_{2}^{(\mathrm{H})}=\frac{1}{\sqrt{2}}\left(\begin{array}{rr}
1 & 1 \\
1 & -1
\end{array}\right),
$$

thus the simple quantum walk on a line is also known as a Hadamard walk. Trivial cases $\eta=0,1$ give oscillatory motion and uniform motion, respectively. The full range of behaviour is obtained by choosing different initial coin states, that is, varying $\alpha, \beta, \phi$ in equation (1).

The quantum walk on the line can be solved analytically in many ways. Straightforward generalisations of methods that work well for classical random walks, such as path counting, and Fourier transformation were both used in Ambainis et al. (2001). We will give examples using these methods in Section 5. Path counting (path integrals) was further refined in Carteret et al. (2003), and a third method using the algebra of the matrix 


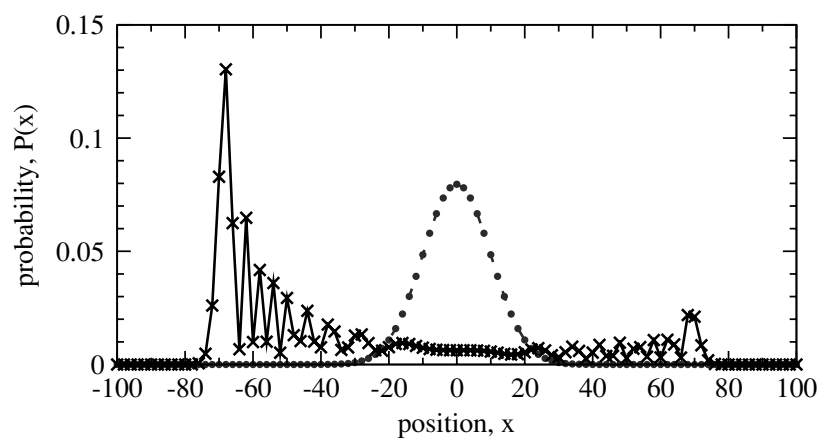

Fig. 1. Comparison of a classical (dots) random walk and a quantum (crosses) walk with initial coin state $|-1\rangle$ and a Hadamard coin operator on a line after 100 steps. Only even points are shown since odd points are unoccupied at even time steps (and vice versa).

operators was presented in Konno (2002; 2005a). A solution using the tools of classical (wave) optics can be found in Knight et al. (2003; 2004). Romanelli et al. (2003) analysed the walk on the line by separating the dynamics into Markovian and interference terms, allowing an alternative method of solution for the long-time limits, which they relate to the dynamics of a kicked rotor. They also show that in the continuum limit one obtains the diffusion equation with added interference terms.

The best way to appreciate the most interesting properties of the solution is in a graph, shown in Figure 1, of the probability distribution of the position of the walker,

$$
P(x, T)=\sum_{c=-1}^{+1}|\langle c \mid \psi(T)\rangle|^{2},
$$

which is obtained in the standard way by tracing out the coin and taking the square modulus of the wavefunction. The quantum walk looks nothing like the classical random walk, it spreads out much faster in a spiky distribution that is a discrete form of an Airey function (Carteret et al. 2003). It is also asymmetric, with the asymmetry determined by the initial coin state. A symmetric distribution can be obtained by choosing the initial coin state as either $(|-1\rangle \pm i|+1\rangle) / \sqrt{2}$, or $\cos (\pi / 8)|-1\rangle-\sin (\pi / 8)|+1\rangle$, see Konno et al. (2004) and Tregenna et al. (2003). The moments have been calculated in Ambainis et al. (2001): for asymptotically large times $T$ for a walk starting at the origin,

$$
\sigma^{2}(T)=\left\langle x^{2}\right\rangle=(1-1 / \sqrt{2}) T^{2},
$$

independent of initial coin state, and

$$
\langle x\rangle_{a}=a(1-1 / \sqrt{2}) T,
$$

where $a \in\{1,-1\}$ is the initial coin state. The standard deviation (from the origin) $\sigma(T)$ is thus linear in $T$, in contrast to $\sqrt{T}$ for the classical walk. Moreover, unlike the classical random walk, the quantum walk evolution depends on the initial state at all subsequent times. 


\subsection{Coined quantum walk on an $N$-cycle: mixing times}

The $N$-cycle is the Cayley graph of the cyclic group of size $N$. It is also a line segment with periodic boundary conditions applied, so there is little extra work to do to apply the solution of the quantum walk on the line to the quantum walk on the cycle. In general, when a quantum walk occurs on the Cayley graph of some group, it is greatly simplified by considering the Fourier space of the particle (Aharonov et al. 2001). On cycles, we are not interested in how far the walker strays from its starting point, but in the mixing properties of the distribution on the cycle. Classical random walks on a cycle mix to a uniform distribution in a time $O\left(N^{2}\right)$. That is, after $O\left(N^{2}\right)$ time steps, the walk has all but forgotten its starting state and is equally likely to be found on any site around the cycle. More formally, we can choose any small $\epsilon$ and find the mixing time $M(\epsilon)$ such that

$$
M(\epsilon)=\min \left\{T \mid \forall t>T:\left\|P(x, t)-P_{u}\right\|_{\text {tv }}<\epsilon\right\}
$$

where $P_{u}$ is the limiting distribution over the cycle, and the total variational distance is defined as

$$
\left\|P(x, T)-P_{u}\right\|_{\mathrm{tv}} \equiv \sum_{x}\left|P(x, T)-P_{u}\right|
$$

In general, any reasonable distance function will do the job here, but we will stick with one commonly used in the literature so that we can make quantitative comparisons. For a classical random walk on a cycle, $M(\epsilon) \sim O\left(N^{2} \log (1 / \epsilon)\right)$.

In the quantum walk on the cycle, the first observation is discouraging: quantum random walks are deterministic, they oscillate forever, and, in general, do not mix even instantaneously. But by defining a time-averaged distribution,

$$
\overline{P(x, T)}=\frac{1}{T} \sum_{t=1}^{T} P(x, t),
$$

quantum walks on cycles do mix. Operationally, this just means randomly choosing a value of $t$ between 1 and $T$, then measuring the position of the quantum walker after $t$ steps. However, unlike the classical random walk, the limiting distribution $\lim \{T \rightarrow \infty\}(\overline{P(x, T)})$ for a quantum walk is not, in general, the uniform distribution. This is in stark contrast with a classical random walk, which always mixes to the uniform distribution (on a regular undirected graph).

Exactly as for $M(\epsilon)$ above, we can then define the mixing time for $\overline{P(x, T)}$, which we will denote $\bar{M}(\epsilon)$,

$$
\bar{M}(\epsilon)=\min \left\{T \mid \forall t>T:\left\|\overline{P(x, t)}-P_{u}\right\|_{\mathrm{tv}}<\epsilon\right\} .
$$

Aharonov et al. (2001) proved that the coined quantum walk on a cycle with an odd number of nodes does mix to the uniform distribution, and has $\bar{M}(\epsilon)$ bounded above by $O\left(\epsilon^{-3} N \log N\right)$, which is almost quadratically faster (in $\left.N\right)$ than a classical random walk. They also proved a lower bound on the time-averaged mixing times for quantum walks on general graphs of bounded degree, suggesting a quadratic improvement over classical random walks is the best that can be achieved. 
Note that there is a price to be paid for the time-averaging: the scaling of the mixing time $\bar{M}(\epsilon)$ depends on $1 / \epsilon$, compared to $\log (1 / \epsilon)$ for a classical random walk (where no time-averaging of $P(x, T)$ is needed). Aharonov et al. (2001) showed that this can be avoided by including an amplification step. The quantum walk is run several times, each time starting from the final state of the previous walk. Applied in the optimal way, their bound on the quantum mixing time reduces to $O(N \log N \log (1 / \epsilon))$. Recent work by Richter improves this result to $O(N \log (1 / \epsilon))$ (Richter 2007a; 2007b). Since the intermediate measurements render the overall quantum walk dynamics non-unitary, we will postpone more detailed discussion to Section 5.5.

This speed up in the rate of mixing of quantum walks is a second key difference between quantum and classical random walks. We can also say a little more about their non-classical limiting distributions. For example, Hadamard walks on cycles with an odd number of nodes converge to the uniform distribution (as was proved in Aharonov et al. (2001)), but those with an even number converge to a non-uniform distribution unless an extra phase $\delta$ is added to the Hadamard coin operator

$$
\mathbf{C}_{2}=\left(\begin{array}{rr}
\sqrt{\eta} & e^{i \delta} \sqrt{1-\eta} \\
e^{-i \delta} \sqrt{1-\eta} & -\sqrt{\eta}
\end{array}\right),
$$

as Tregenna et al. (2003) and Bednarska et al. (2003) show. By an appropriate choice of coin operator, a walk on any size cycle can be made to converge to either a uniform or non-uniform probability distribution. In classical random walks, the properties of the limiting distribution depend solely on the form of the graph.

\subsection{Periodicity in coined quantum walks on cycles}

A further curious property of quantum walks on cycles is that for a few special choices of cycle size $N$, a perfectly periodic walk arises where the quantum walk returns exactly to its starting state after a fixed number of steps $\Omega$, and then repeats the sequence over again, returning at $2 \Omega$, and so on. The classical random walk has no such behaviour and returns to its initial state only after an irregular numbers of steps. This periodicity is not connected with whether the limiting distribution is uniform or not, since here we are concerned with exact return to the initial state, rather than the time-averaged quantity in equation (14). Some of these periodic walks also mix instantaneously, if we allow a walk on an even cycle to be considered mixed on just the odd or just the even-numbered sites (the same issue arises when considering classical random walks on even-sized cycles).

Using a Hadamard coin, the 'cycle' of size $N=2$ is trivially periodic, returning to its original state after two steps. A cycle of size $N=4$ has a period of eight steps. This was first noted in Travaglione and Milburn (2002). Tregenna et al. (2003) continued this investigation, and found the cycle with $N=8$ has a period of 24 steps, but $N=16$ is chaotic, and does not return to its initial state exactly, even after many thousands of steps. These periodic cycles also exhibit instantaneous mixing half way through on their way to returning to the initial state, but instantaneous mixing has not been systematically studied in discrete walks on the cycle. If the coin is allowed to be biased, a few more 
periodic examples can be found, $N=6$ with period 12 , and $N=10$ with period 60 . With judicious choice of phases in the coin operator, $N=3$ has a period of 12 , and $N=5$ has a period of 60 , which is clearly related to $N=6$ and $N=10$, respectively, but these were the only periodic odd- $N$ cycles found. It is not known whether all periodic quantum walks on a cycle have been identified, but Tregenna et al. (2003) conjectures that there are only a finite number of such solutions and that this is nearly all if not all of them. Neither periodicity nor instantaneous mixing has been explored on more general graphs beyond the trivial extensions of the above to the torus and twisted torus in Tregenna et al. (2003).

\subsection{Coined quantum walks on higher dimensional graphs}

Classical random walks are not limited to one-dimensional structures, and neither are quantum walks. All that is required for the discrete-time quantum walk is a coin that is large enough to handle the number of choices at each vertex the quantum walker might land on.

Consider a general graph $G$, with $N$ vertices in a set $V$, connected by edges from the set $E$. How one proceeds depends on what prior knowledge one has about $V$ and $E$. Since we are just discussing undirected graphs, if vertices $x, y \in V$ are connected by an edge $e_{x y} \in E$, then $e_{y x} \in E$ also, and one is allowed to travel both from $x$ to $y$ and from $y$ to $x$. The usual way to represent the structure of the graph is in an adjacency matrix $\mathbf{A}$, which has unit entries for each $A_{x y}$ for which $e_{x y} \in E$, and zeros everywhere else. Since $A_{x y}=A_{y x}$ for an undirected graph, $\mathbf{A}$ is symmetric. This representation of the graph assumes that $e_{x y}$ is unique, that is, there is at most one edge between any two vertices in G. Classically, one can subsume multiple edges between the same vertices into a set of 'edge weights'. However, a quantum walker might traverse both edges at once but with different phases, which would, in general, have a different outcome to one weighted edge.

Given no further information about $G$, there could be as many as $N(N-1) / 2$ edges, corresponding to the complete graph, for which every vertex has $N-1$ edges leading from it. In this case the coined quantum walk needs to use a coin of at least this size $(N-1)$, the details of how to implement this quantum walk may be found in Kendon (2006a). If we know the maximum degree of the graph, $d$, we only need a coin of size $d$. The details of how to implement this quantum walk, originally from Watrous (2001), are more accessibly described in Ambainis (2004) and Kendon and Sanders (2004). A different approach, using self-loops to make the degree of the graph constant, is mentioned in Kempe (2003a).

Here we summarise the approach given in Kendon and Sanders (2004). First we define our Hilbert space, $\mathscr{H}_{\mathrm{vc}}$, for the quantum walk. This contains the $N$-dimensional Hilbert space

$$
\mathscr{H}_{\mathrm{v}}=\operatorname{span}\left\{|x\rangle_{\mathrm{v}}: x \in \mathbb{Z}_{N},{ }_{\mathrm{v}}\left\langle x \mid x^{\prime}\right\rangle_{\mathrm{v}}=\delta_{x x^{\prime}}\right\} \subset \mathscr{H}_{\mathrm{vc}}
$$

of vertex states, and, for the coin, a $d$-dimensional Hilbert space

$$
\mathscr{H}_{\mathrm{c}}=\operatorname{span}\left\{|c\rangle_{\mathrm{c}}: c \in \mathbb{Z}_{d}, \mathrm{c}\left\langle c \mid c^{\prime}\right\rangle_{\mathrm{c}}=\delta_{c c^{\prime}}\right\}
$$


where $d$ is the degree of the graph. The basis states of $\mathscr{H}_{\mathrm{vc}}$ are given by

$$
\mathscr{B}_{\mathrm{vc}}=\left\{|x, c\rangle \equiv|x\rangle_{\mathrm{v}}|c\rangle_{\mathrm{c}} ; x \in \mathbb{Z}_{N}, c \in \mathbb{Z}_{d}\right\}
$$

with cardinality $N d$. For a basis state $|x, c\rangle$, the index $x$ identifies the vertex number and $c$ the $c^{\text {th }}$ state of the coin. For an edge $e_{x x^{\prime}}$, we associate the coin state $c$ with the edge at $v_{x}$, and the coin state $c^{\prime}$ with the other end of the edge at $v_{x^{\prime}}$. The values of $c$ and $c^{\prime}$ are arbitrary, but fixed throughout the quantum walk to ensure the quantum walker traverses the the graph in a consistent manner. We define the mapping

$$
\zeta: \mathbb{Z}_{N} \times \mathbb{Z}_{d} \rightarrow \mathbb{Z}_{N} \times \mathbb{Z}_{d}:(x, c) \mapsto \zeta(x, c)=\left(x^{\prime}, c^{\prime}\right),
$$

where $(x, c)$ and $\left(x^{\prime}, c^{\prime}\right)$ label each end of $e_{x x^{\prime}}$.

The unitary quantum walk evolves by repetition of two steps: a coin toss and a conditional swap. The coin operator

$$
C: \mathscr{H}_{\mathrm{vc}} \rightarrow \mathscr{H}_{\mathrm{vc}}:|x, c\rangle \mapsto \sum_{\tilde{c} \in \mathbb{Z}_{d}} C_{\tilde{c} \tilde{c}}^{x}|x, \tilde{c}\rangle_{\mathrm{c}}
$$

is a block diagonal matrix with each block labelled by $x$. The $x$-dependence of the coin matrix allows sufficient freedom in the quantum walk dynamics for the quantum coherence properties of the coin to vary between vertices, for vertices to act as origins and endpoints, and for vertices to have different degrees from each other. If $v_{x}$ has degree $d_{x}<d$, we require $C_{\tilde{c} \tilde{c}}^{x}=0$ for all $\tilde{c}$ values not used to label an edge at $v_{x}$. This restricts the coin operator so that it only produces states that have a valid mapping under $\zeta$. If

$$
C_{c \tilde{c}}^{x}=C_{c \tilde{c}}^{x^{\prime}} \forall x, x^{\prime},
$$

we have the special case of a fixed degree graph where the coin operator is identical for all vertices, as in the walk on a line or cycle described in the previous sections.

The unitary conditional swap operator is given by

$$
\mathbf{S}: \mathscr{H}_{\mathrm{vc}} \rightarrow \mathscr{H}_{\mathrm{vc}}:|x, c\rangle \mapsto\left|x^{\prime}, c^{\prime}\right\rangle
$$

which updates the position of the walker and the coin state according to the mapping $\zeta$ in equation (20), that is, it moves the walker and coin to the vertex $v_{x^{\prime}}$ along edge $e_{x x^{\prime}}$. We note that by our stipulation that $c$ and $c^{\prime}$ label opposite ends of $e_{x x^{\prime}}$, it follows that $\mathbf{S}=\mathbf{S}^{-1}$, and is thus unitary, as required for quantum evolution. The sequence of a coin flip and a conditional swap is a transition over the unit time step, which we denote by unitary $\mathbf{U}=\mathbf{S C}$. The quantum walk can then be written

$$
|\psi(t)\rangle=\mathbf{U}^{t}|\psi(0)\rangle,
$$

where $|\psi(t)\rangle$ and $|\psi(0)\rangle$ can be expressed in basis states as per equation (4).

\subsection{Coined quantum walks on regular lattices}

Generally, we can only find analytical solutions of equation (24) for special graphs of fixed degree and high symmetry (see, for example, Grimmett et al. (2004)). The scattering theory methods of Feldman and Hillery (2004) are worth noting as a possible exception, though 
few applications have been presented. Numerical studies are less constrained, due to the simplicity of the step-by-step evolution of quantum walks. Examples of quantum walks on various graphs of degree larger than two can be found in Mackay et al. (2002), Tregenna et al. (2003) and Carneiro et al. (2005), and in the quantum walk search algorithm in Shenvi et al. (2003) - see Section 4.1. Many of the basic results for quantum walks on the line and cycle, such as faster spreading, carry over to higher dimensional graphs. This was shown numerically for regular lattices of degree three and four in Mackay et al. (2002). However, higher dimensional coins have a much wider set of possible types of coin operators, and further numerical study (Tregenna et al. 2003) shows that the quantum speed up is not automatic for all possible choices.

The role of lattice symmetry combined with the symmetry in the dynamics of the quantum walk in determining the spreading has been further studied and clarified in Krovi and Brun (2006a; 2006b). Most of the examples studied to date have a high degree of symmetry, and it makes sense to choose a quantum coin operator that reflects the symmetry in the problem. Two examples are worth noting. First, the DFT (discrete Fourier transform) coin operator is unbiased, but asymmetric in that you cannot interchange the labels on the directions without changing the coin operator. First used in Mackay et al. (2002), for $d=3$ it looks like

$$
\mathbf{C}_{3}^{(D)}=\frac{1}{\sqrt{3}}\left(\begin{array}{ccc}
1 & 1 & 1 \\
1 & e^{i \omega} & e^{-i \omega} \\
1 & e^{-i \omega} & e^{i \omega}
\end{array}\right)
$$

where $e^{i \omega}$ and $e^{-i \omega}$ are the complex cube roots of unity, For $d=2$, the DFT coin reduces to the Hadamard coin, equation (8), though this is not the only way to generalise the Hadamard coin to higher dimensions (see, for example, Tregenna et al. (2003) and Tadej and Życzkowski (2006)). Quantum walks using the DFT coin operator have interesting non-classical properties, which have been studied in Mackay et al. (2002), Tregenna et al. (2003), Carneiro et al. (2005) and Krovi and Brun (2006a), but none of them have yet provided any quantum algorithms.

The second commonly used coin operator is a highly symmetric coin based on Grover's diffusion operator (Grover 1996) with elements $2 / d-\delta_{i j}$, which in matrix form for $d=3$ is

$$
\mathbf{C}_{3}^{(G)}=\frac{1}{3}\left(\begin{array}{rrr}
-1 & 2 & 2 \\
2 & -1 & 2 \\
2 & 2 & -1
\end{array}\right)
$$

The Grover coin is biased but symmetric, it is the symmetric unitary operator furthest from the identity. It was first used in quantum walks in Watrous (2001), and is the key ingredient in the quantum walk searching algorithm in Shenvi et al. (2003) - see Section 4.1. Inui et al. (2004) studied the localisation properties related to searching on a two-dimensional lattice. Szegedy (2004a; 2004b) introduced a generalisation of the Grover coin that quantises an arbitrary Markov chain: essentially this allows for edge weights on the graph, and works for graphs of variable degree as well as regular graphs. 


\subsection{Coined quantum walk on the hypercube: hitting times}

In one of the few analytical studies of coined quantum walks in higher dimensions, Moore and Russell (2002) used a Grover coin for the hypercube, which has $2^{n}$ vertices each of dimension $d=n$. The n-dimensional hypercube is the Cayley graph of $\mathbb{Z}_{2}^{n}$, so the solution follows the same general method as for the line and the $N$-cycle, using Fourier transformation of the position space. Moore and Russell (Moore and Russell 2002) determined from this solution that the discrete-time quantum walk on the hypercube of size $n$ has approximate instantaneous mixing times:

$$
M_{\text {inst }}(\epsilon)=\left\{t \quad: \quad\left\|P(x, t)-P_{u}\right\|_{t v}<\epsilon\right\}
$$

for $t=n k \pi / 4$ for all odd $k>0$ and $\epsilon=O\left(n^{-7 / 6}\right)$. This is an improvement over a classical random walk, which mixes in time $O(n \log n)$, but requires measurement of the quantum walk at exactly the right time (otherwise the walk 'unmixes' again as it proceeds). They also consider $\overline{P(x, t)}$, and find, using the methods of Aharonov et al. (2001), that $\bar{M}(\epsilon)$ is exponentially large, $O\left(2^{n}\right)$.

These results for mixing times are discouraging, but there is another useful property one can test on a hypercube: hitting times. Kempe (2003b; 2005) proved that a quantum walk can travel from one corner of a hypercube to the opposite corner exponentially faster than a classical random walk. Kempe defines two different hitting times: instantaneous, where one measures the destination corner at the optimal time; and concurrent, where one checks the destination after each step to see if the walker has arrived yet. This gives us our first taste of non-unitary evolution in a quantum walk, since measuring the destination node at each step destroys some of the coherences in the quantum state. Recent work in Krovi and Brun (2006a) expands on these ideas, and we will describe them in more detail in Section 5.6. There are other classical algorithms that can cross a hypercube efficiently, so it does not provide a quantum algorithm with a true advantage over classical ones.

\section{Continuous-time quantum walks}

Continuous-time quantum walks on a discrete lattice have their origins as far back as Feynman et al. (1964). Their use for quantum algorithms was first suggested in Farhi and Gutmann (1998), which showed numerically that they can reach the ends of certain network configurations more efficiently than classical random walks. A proven exponential speed up in a quantum algorithm using a continuous-time quantum walk came a few years later in Childs et al. (2003), which we will briefly describe in Section 4.2. In this section we will first describe the continuous-time quantum walk dynamics on a general graph, then compare it (numerically) with the discrete-time walk on the line. We will then give two further examples, on cycles and hypercubes, that we will need later.

\subsection{Continuous-time quantum walks on general graphs}

The continuous-time quantum walk naturally works on any undirected graph. Farhi and Gutmann (1998) simply used the adjacency matrix A, which is symmetric for an undirected 
graph and therefore Hermitian, to form the Hamiltonian for the evolution of the quantum state:

$$
i \frac{d}{d t}\langle x \mid \psi(t)\rangle=\sum_{y}\langle x|\mathbf{H}| y\rangle\langle y \mid \psi(t)\rangle .
$$

Here $\mathbf{H}=\gamma \mathbf{A}$, where $\gamma$ is the hopping rate per edge per unit time, and $|\psi(t)\rangle$ is now a vector in the Hilbert space of position (vertices in the graph) only (no coin). Continuoustime quantum walks achieve what was impossible without a coin in a discrete-time quantum walk: the traversing of all possible paths in superposition. The formal solution of equation (28) is

$$
|\psi(t)\rangle=e^{-i \gamma \mathbf{A} t}|\psi(0)\rangle .
$$

On the same graph, a continuous-time classical random walk evolves as

$$
\frac{d}{d t} P(x, t)=\gamma \sum_{y}\left\{A_{x y} P(y, t)-A_{y x} P(x, t)\right\} .
$$

Comparing equation (28) with equation (30) shows that in the quantum walk, the second term, which is necessary for the conservation of probability, is missing. We only need a Hermitian operator for quantum evolution, and since $A_{x y}=A_{y x}$ guarantees this, we are free to examine this dynamics as well as that obtained in more direct analogy to equation (30) by using the Laplacian,

$$
i \frac{d}{d t}\langle x \mid \psi(t)\rangle=\gamma \sum_{y}\{\langle x|\mathbf{A}| y\rangle\langle y \mid \psi(t)\rangle-\langle y|\mathbf{A}| x\rangle\langle x \mid \psi(t)\rangle\} .
$$

For graphs where all the vertices are of the same degree $d$, the Hamiltonian in equation (31) becomes $\mathbf{H}=\gamma(\mathbf{A}-d \mathbb{1})$, and the solution to this can be written

$$
|\psi(t)\rangle=e^{-i \gamma(\mathbf{A}-d \mathbb{1}) t}|\psi(0)\rangle .
$$

Since A commutes with the identity, the two terms in the exponential can be factored, giving

$$
|\psi(t)\rangle=e^{-i \gamma \mathbf{A} t} e^{i \gamma d \mathbb{1} t}|\psi(0)\rangle .
$$

The factor $e^{i \gamma d} \mathbb{1}_{t}$ is only an irrelevant global phase, which makes no difference to observable quantities if omitted (Ahmadi et al. 2003), thus equation (33) is equivalent to equation (29) for graphs of fixed degree. For graphs of general degree, however, the dynamics with the second term included will have a different evolution, as Childs and Goldstone (2004a) pointed out. So far, only graphs of fixed degree have been studied in any detail in the literature, so the differences between the two versions have not been explored.

Equation (29) looks remarkably similar to the discrete case, equation (24): both can be written in the form

$$
|\psi(t)\rangle=\mathbf{U}^{t}|\psi(0)\rangle
$$

with unitary operator $\mathbf{U}=\mathbf{S C}$ for the discrete-time walk and $\mathbf{U}=e^{-i \gamma \mathbf{A}}$ for the continuoustime walk. But, unlike the classical case, where the limit of the discrete-time walk as the time step goes to zero can be taken in a way that gives the continuous-time walk, in the quantum case the similarity is deceptive. The discrete and continuous walks have 


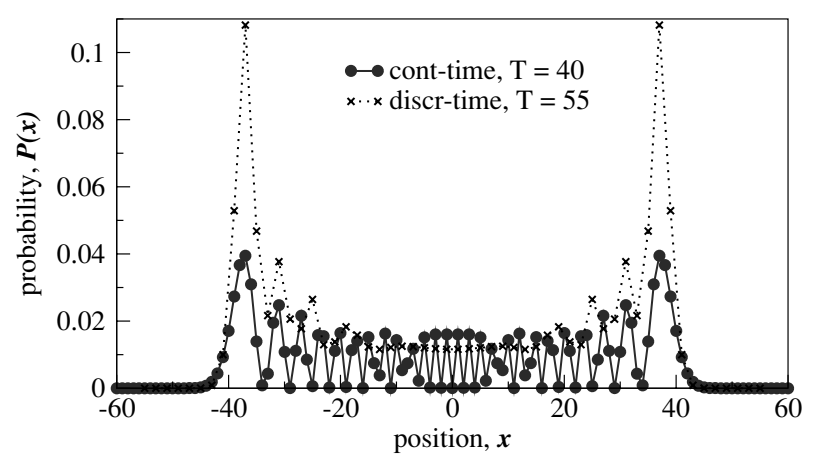

Fig. 2. Comparison of continuous-time (dots) and discrete-time (crosses) quantum walks on a line starting at the origin after 40 and 55 steps, respectively. The discrete-time walk has initial coin state $(|-1\rangle+i|1\rangle) / \sqrt{2}$, and only even points are shown since odd points are unoccupied at even time steps (and vice versa). All points are shown for the continuous-time walk, which has hopping rate $\gamma=0.5$.

Hilbert spaces of different sizes, since the continuous-time quantum walk has no coin: the continuous-time walk therefore cannot be the limit of the discrete-time walk unless it can be restricted to a subspace that excludes the coin. Strauch $(2006 \mathrm{~b} ; 2006 \mathrm{a})$ show how to do this for the walk on the line: it turns out that the appropriate limit of the discrete time quantum walk produces two copies of the continuous-time quantum walk, one for each of the two coin degrees of freedom. Alternatively, if one adds a coin space to the continuous-time quantum walk, a quantum dynamics is obtained that is more obviously the continuum limit of the discrete-time coined quantum walk, see Szegedy (2004b) and Childs and Goldstone (2004b). The quantised Markov chain formalism introduced in Szegedy (2004a) is yet another convenient way of constructing quantum walks in a parallel manner to classical random walks - see, for example, Weiss (1994). The stochastic matrix governing the Markov chain corresponds to the adjacency matrix in the graph-based description above.

\subsection{Continuous-time quantum walk on the line}

A comparison of the simple case of the walk on the line will show us the main similarities and differences between discrete-time and continuous-time quantum walks. Equation (28) has a straightforward solution in terms of Bessel functions,

$$
|\psi(t)\rangle=\sum_{x=-\infty}^{+\infty}(-i)^{x} J_{x}(t)|x\rangle,
$$

where $J_{x}(t)$ is the Bessel function of order $x$. Using a hopping rate of $\gamma=0.5$ so that the total hopping probability per unit time is one, after 40 units of time we get the probability distribution shown in Figure 2. The discrete-time quantum walk evolved for 55 time steps is also shown for comparison. We can see that the shapes of the distribution are comparable, the difference in the height of the peaks is due largely to the continuous-time 
walk having support on both odd and even sites, while the discrete-time walk is restricted to sites with the same parity as the time step. Nonetheless, the continuous-time walk has a very oscillatory nature, especially in the central region. The two types of walks propagate at somewhat different speeds, as evidenced by the different time instants at which they have the same width. Both spread linearly, but with different constant prefactors, so both show a quadratic speed up in their spreading compared to classical random walks. For the continuous-time quantum walk starting at the origin, for asymptotically large times $T$,

$$
\sigma^{2}(T)=\left\langle x^{2}\right\rangle=T^{2} / \gamma
$$

The continuous-time quantum walk on the line is always symmetric if the hopping rate is the same for both directions, this is the main difference compared with the discrete-time walk, where the coin generally skews the walk unless special choices are made for the initial state.

\subsection{Continuous-time quantum walk on an $N$-cycle and other circulant graphs}

The continuous time quantum walk on an $N$-cycle is straightforward to analyse. The adjacency matrix $\mathbf{A}$ of the $N$-vertex cycle graph is a circulant matrix, it has eigenvalues $\lambda_{x}=2 \cos (2 \pi x / N)$ with corresponding eigenvectors $\left|b_{x}\right\rangle$, where

$$
\left\langle y \mid b_{x}\right\rangle=\frac{1}{\sqrt{N}} \exp (-2 \pi i x y / N)
$$

for $x=0,1, \ldots, N-1$. Taking the initial state of the quantum walk to be $|\psi(0)\rangle=|0\rangle$, we can solve $|\psi(t)\rangle=e^{-i t \mathbf{H}}|0\rangle$ by decomposing $|0\rangle$ in terms of the eigenvectors $\left|b_{x}\right\rangle$, giving

$$
|\psi(t)\rangle=\frac{1}{\sqrt{N}} \sum_{x=0}^{N-1} e^{-i t \lambda_{x}}\left|b_{x}\right\rangle .
$$

One can apply the same notions of mixing times to a continuous-time quantum walk as for the discrete-time walk. Like the discrete-time quantum walk on a cycle, the probability distribution $P(x, t)$ of the continuous-time walk does not mix asymptotically, and is known to have exact instantaneous mixing for only a few special cases, $N=3$ and $N=4$ (Ahmadi et al. 2003), that is, even fewer cases than are known for the discrete-time walk on the cycle as mentioned in Section 2.3. We can define the continuous-time version of equation (14), to give the average probability

$$
\overline{P(x, T)}=\frac{1}{T} \int_{0}^{T} d t P(x, t)
$$

where $P(x, t)=|\langle x \mid \psi(t)\rangle|^{2}$. While this does mix asymptotically, in general, like the discretetime walk, the limiting distribution is not uniform: it retains one or more peaks reflecting the initial state. Note that the properties of continuous-time walks on cycles are not dependent on whether the cycle has an odd or even number of nodes, unlike the discretetime quantum walk.

Adamczak et al. (2003) and Adamczak et al. (2007) showed that continuous-time walks on cycles are nearly uniform mixing, that is, if one relaxes the condition on how well they 
approach the uniform distribution from an arbitrarily small $\epsilon$, they satisfy

$$
\bar{M}(1 / 4 N)=\min \left\{T \mid \forall t>T:\|P(x, t)-1 / N\|_{t v} \leqslant \frac{1}{4 N}\right\} \sim O(N) .
$$

Adamczak et al. extended their analysis of mixing in continuous-time quantum walks to a wider variety of circulant graphs, this was continued in Lo et al. (2006): mixing to non-uniform distributions turns out to be the norm for these types of graphs. Carlson et al. (2006) identifies graphs where, with the use of edge weights, any distribution on the graph can be obtained (universal mixing). Carlson et al. also identified another class of graphs that are instantaneous uniform mixing - the claw or star graphs.

\subsection{Continuous-time quantum walk on the hypercube}

Our next example of a continuous-time quantum walk is on an $n$-dimensional hypercube. Moore and Russell (2002) gave an analytical solution for the continuous-time quantum walks on the hypercube as well as for the discrete-time walk (see Section 2.4). The analysis for the continuous-time walk makes use of the hypercube's product graph structure. We label the vertices with $n$-bit strings, with edges connecting those pairs of vertices that differ in exactly one bit. Then, using the Pauli matrix, $\sigma_{x}$, which is the bit flip operator,

$$
\sigma_{x}=\left(\begin{array}{ll}
0 & 1 \\
1 & 0
\end{array}\right)
$$

the adjacency matrix can be decomposed into the sum

$$
\mathbf{A}=\sum_{j=1}^{n} \mathbb{1} \otimes \cdots \otimes \sigma_{x} \otimes \cdots \otimes \mathbb{1},
$$

where the $j$ th term in the sum has $\sigma_{x}$ as the $j$ th factor in the tensor product. Each term thus flips the bit in the vertex label, which corresponds to traversing the edge to the appropriately labelled neighbouring vertex. Using $\mathbf{H}=\gamma \mathbf{A}$ for the quantum walk, we have ${ }^{\dagger}$

$$
\begin{aligned}
& \mathbf{U}^{t}=e^{-i \mathbf{H} t} \\
& =\prod_{j=1}^{n} \mathbb{1} \otimes \cdots \otimes e^{-i \gamma t \sigma_{x}} \otimes \cdots \otimes \mathbb{1} \\
& =\left[e^{-i \gamma t \sigma_{x}}\right]^{\otimes n} \\
& =\left(\begin{array}{ccc}
\cos (\gamma t) & -i \sin (\gamma t) \\
-i \sin (\gamma t) & \cos (\gamma t)
\end{array}\right)^{\otimes n} .
\end{aligned}
$$

Applying $\mathbf{U}^{t}$ to the initial state $|\psi(0)\rangle=|0\rangle^{\otimes n}$, we have

$$
\mathbf{U}^{t}|\psi(0)\rangle=[\cos (\gamma t)|0\rangle-i \sin (\gamma t)|1\rangle]^{\otimes n},
$$

which corresponds to a uniform state exactly when $\gamma t$ is an odd multiple of $\frac{\pi}{4}$. The choice

\footnotetext{
$\dagger$ Moore and Russell (2002) used $U=e^{i H}$, which is just the complex conjugate and makes no difference to observable quantities.
} 
of $\gamma$ is thus pivotal for making a fair comparison of the instantaneous mixing time. Moore and Russell (2002) chose $\gamma=1 / n$ so that the total probability of making a hop to any neighbouring site per unit time is unity. With this choice, the continuous-time quantum walk mixes exactly instantaneously in time $O(n)$, which is a logarithmic improvement over $O(n \log n)$ for the mixing time of a classical random walk. They also showed that for the time-averaged mixing time given by equation (15), the continuous-time walk never mixes. These results can be compared with the discrete-time walk on the hypercube, which has approximate instantaneous mixing (instead of exact), and $\bar{M}(\epsilon)$ mixing in exponential time.

The continuous-time quantum walk on the hypercube also hits the opposite corner in linear time, which is exponentially faster than a classical random walk: we will obtain this result in Section 6.3 as a special case of the calculation of the effects of decoherence. As with the coined quantum walk, this exponentially fast hitting time is highly dependent on the symmetry. Keating et al. (2006) applied results from localisation theory (Anderson 1958) to argue that this behaviour is exceptional, and the norm on less regular graphs is a quantum walk that tends to stay near its starting state.

\section{Algorithms using quantum walks}

Starting with Shor's algorithm for factoring large numbers (Shor 1997), many of the quantum algorithms found so far belong to the same family, and are based on the use of Fourier transforms to identify a hidden subgroup (Lomont (2004) provides a recent review). This works well (that is, can be exponentially better than known classical methods) for Abelian groups, but extending the method to non-Abelian groups, where some of the notorious hard problems, such as graph isomorphism, lie, is proving tricky. An obvious place to look for new ideas is where classical algorithms are having the most success to see if a quantum version could be even faster. Randomised algorithms are one such arena, providing the best known algorithms for approximating the permanent of a matrix (Jerrum et al. 2001), finding satisfying assignments to Boolean expressions ( $k$ SAT with $k>2$ ) (Schöning 1999), estimating the volume of a convex body (Dyer et al. 1991), and graph connectivity (Motwani and Raghavan 1995). Classical random walks also underpin many standard methods in computational physics, such as Monte Carlo simulations. Expanding the repertoire of methods for quantum algorithms was the motivation behind the recent upsurge of interest in quantum walks. The first proper algorithms using quantum walks appeared in Childs et al. (2003) and Shenvi et al. (2003), and more have followed since: for a short survey, see Ambainis (2003). We will briefly describe the first two quantum walk algorithms, since they are of two distinct generic types, and later algorithms are mostly variants of the quantum walk search outlined in the next section.

\subsection{Quantum walk searching}

Quadratically faster spreading, as described in Section 2.1, is not a quantum algorithm, but quantum searching of an unsorted database (for example, finding the name corresponding to a given number by searching a telephone directory) is the reverse process: start in a 


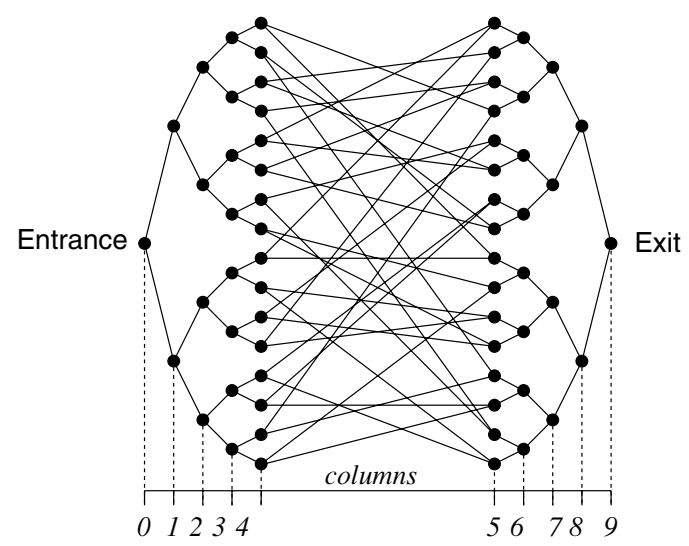

Fig. 3. 'Glued trees' graph used in the algorithm of Childs et al. (2003). The example shown is for $N=4$, with $2 N+2=10$ columns labelled at the bottom of the figure, and $2\left(2^{(N+1)}-1\right)=62$ nodes. The task is to travel from the entrance to the exit without getting lost in the randomly joined middle section of the graph. The gap between columns 4 and 5 is for clarity in the figure and is not significant in the algorithm.

uniform superposition over the whole database and home in on the marked item. The first quantum algorithm for this problem was given in Grover (1996), using a method of amplitude amplification, from which a quadratic speed up over classical searching is obtained. It can be shown that a quadratic speed up is the best possible improvement for this problem (Bennett et al. 1997). A classical search of an unsorted database potentially has to check all $N$ entries in the database, and on average has to check at least half. A quantum search only needs to make $O(\sqrt{N})$ queries, though the queries ask for many answers in superposition. Shenvi et al. (2003) showed that a quantum walk can also search an unsorted database with a quadratic speed up. They represent the database by the vertices of a graph with a regular structure (lattice, hypercube...) and start with the quantum walker in a superposition of all positions on the vertices. The quantum walk proceeds using a Grover coin operator of appropriate dimension at every vertex except the marked item. For the marked item, almost any other coin operator can be used, this is enough to break the symmetry of the quantum walk and cause the walker to converge on the marked vertex in $\sim \pi / 2 \sqrt{N / 2}$ steps.

Since then, several variations on quantum searching for a single item have been analysed, all essentially searching for a set of marked items of some sort (Magniez et al. 2005; Childs and Eisenberg 2005; Ambainis 2004). Spatial searching, where moving from one item in the database to the next is also counted as a cost, is also faster with a quantum algorithm, and, curiously, the continuous-time walk finds this a little harder than the discrete-time walk: in low dimensions it needs a coin too (Childs and Goldstone (2004a; 2004b).

\section{2. 'Glued trees' algorithm}

Childs et al. (2003) proved that a continuous-time quantum walk could produce a result exponentially faster than any classical algorithm when finding a route across a particular sort of network: see Figure 3. This is a rather artificial problem, but proves in principle 
that quantum walks are a powerful tool. The task is to find your way from the entrance node to the exit node, treating the rest of the network like a maze where you cannot see the other nodes from where you stand, only the choice of paths. It is easy to tell which way is forward until you reach the random joins in the centre. After this, any classical attempt to pick out the correct path to the exit gets lost in the central region and takes exponentially long, on average, to find the way out. A quantum walk, on the other hand, travels through all the paths in superposition, and the quantum interference between different paths allows the quantum walker to figure out which way is forward right up to the exit, which it finds in time proportional to the width of the network. The proof is quite technical, involving oracles and consistent colourings of the network, and requires the simulation of a continuous-time quantum walk on a discrete quantum computer. We refer the reader to the original paper (Childs et al. 2003) for the details, since we do not need them here for our discussion of decoherence.

Discrete and continuous-time quantum walks are generally expected to have the same computational power. They give broadly similar results for algorithmic properties such as mixing times and hitting times in those cases where both forms have been applied to the same problem, with the possible exception of spatial search (Childs and Goldstone 2004a), which, as noted above, requires a coin for maximum efficiency even in the continuoustime walk in low dimensions (Childs and Goldstone 2004b). For a discussion of how both discrete and continuous-time quantum walk implementations would use roughly the same computational resources for the 'glued trees' problem, see Kendon (2006a).

\section{Decoherence in coined quantum walks}

As we have seen, while there are many similarities between discrete and continuous-time quantum walks, there are also some distinctly different behaviours, and this turns out to be true also under the influence of decoherence. We will thus devote a separate section to each type, starting here with coined quantum walks and following in Section 6 with continuous-time quantum walks. We are going to take a very broad view of decoherence in this review as any dynamics that tends to remove the quantum coherences in some way, be it unwanted (as in environmental decoherence in an experimental system), intentional (to tune the properties of the quantum walk), or a byproduct of some other operation, such as measurement. One of the earliest uses of non-unitary quantum walks, in Aharonov et al. (1992), was measurement-based.

Quantum walks are a very broad class of quantum dynamics, with overlap into related areas such as quantum graphs (Kottos and Smilansky 1997). One way to justify a particular quantum dynamics as being a 'quantum walk' is to see if it turns into a classical random walk when decohered. Since classical random walks are also a very broad class of dynamics, this gets reasonably close to a workable definition of a quantum walk, at least for the discrete-time case. Many early studies of quantum walks took the trouble to show numerically that, for specific cases, their quantum walks decohered into classical random walks (see, for example, Mackay et al. (2002), where the coin is dephased in the study of a quantum walk on the line). A more systematic treatment of the quantum to classical transition in a general quantum walk appears in Kendon and Sanders (2004), which emphasises the importance of (a) demonstrating that quantum walks exhibit both wave 
(pure quantum) and particle (decohered) dynamics, and (b), for a non-unitary quantum walk, being able to interpolate between these two modes of behaviour by turning the decoherence up or down. Košík et al. (2006) explicitly calculated the cases of Grover and DFT coins on a Cartesian lattice $\left(\mathbb{Z}^{d}\right)$, with random phase shifts applied to the coin to induce decoherence rather than measurements. They show that the resulting distributions match the expected classical random walks (which may be biased if the initial state or coin operator is biased, $c f$. Section 2.1).

Decoherence is usually modelled as a non-unitary evolution of the quantum walk, so we will need some extra formalism for mixed states using density matrix operators. Following the notation in Section 2.4, the (time-dependent) density operator

$$
\boldsymbol{\rho}=\sum_{x, c} \sum_{x^{\prime}, c^{\prime}} \rho_{x c, x^{\prime} c^{\prime}}|x, c\rangle\left\langle x^{\prime}, c^{\prime}\right|
$$

is a positive ( $\boldsymbol{\rho}=\boldsymbol{\rho}^{\dagger}$ with positive real spectrum), unit-trace, bounded linear operator on $\mathscr{H}_{\mathrm{vc}}$, in the basis $\mathscr{B}_{\mathrm{vc}}-$ see equation (19). In the decomposition of $\rho$ into the computational basis, the rows and columns of $\rho$ are indexed by $x c$ and $x^{\prime} c^{\prime}$, which run over the position states and coin states of the Hilbert space. The state is pure if and only if $\rho^{2}=\rho$. A typical initial condition is $\boldsymbol{\rho}(t=0)=|0,0\rangle\langle 0,0|$, corresponding to the walker starting at vertex $v_{0}$ carrying a coin in the state labelled zero. In general, the density operator is mapped to a new density operator via a completely positive (CP) map

$$
\mathscr{U}: \rho \mapsto \mathscr{U} \rho
$$

The CP map $\mathscr{U}$ performs both the coin flip and the conditional swap over one time step. More explicitly, we can write

$$
\mathscr{U} \boldsymbol{\rho}=\sum_{i \in \Theta} \mathbb{U}_{j}^{\dagger} \boldsymbol{\rho} \mathbb{U}_{j} \quad \sum_{i \in \Theta} \mathbb{U}_{j}^{\dagger} \mathbb{U}_{j}=\mathbb{1},
$$

with $j$ an index of non-unitary evolutionary 'instances' and $\mathbb{U}_{j}$ the corresponding Kraus operator. These instances may be discerned by a measurement record, with $j$ the record index. The cardinality of $\Theta$ can be finite, countably infinite, or even uncountable. In the case of unitary evolution, $\Theta$ has a cardinality of one, so there is a single, unitary $\boldsymbol{U}$ for which $\boldsymbol{\rho} \mapsto \mathscr{U} \boldsymbol{\rho}=\boldsymbol{U} \boldsymbol{\rho} \boldsymbol{U}^{\dagger}$. Unitary quantum walk evolution can be expressed as

$$
\boldsymbol{\rho}(t)=\mathscr{U}^{t} \boldsymbol{\rho}(0), \mathscr{U} \equiv \mathscr{S} \mathscr{C}, \mathscr{S} \mathscr{C} \boldsymbol{\rho} \equiv \boldsymbol{S C} \boldsymbol{\rho} \boldsymbol{C}^{\dagger} \boldsymbol{S}^{\dagger},
$$

where, for the discrete-time walk, we assume $t \in \mathbb{Z}$. Thus, for the unitary walk, a single step is given by $\boldsymbol{U}=\boldsymbol{S} \boldsymbol{C}$, while for the non-unitary walk we can either add an extra non-unitary operation (measurement) $\left\{\mathbb{P}_{j}\right\}$,

$$
\mathscr{U}: \rho \mapsto \sum_{i \in \Theta} \mathbb{P}_{j} S C \rho C^{\dagger} S^{\dagger} \mathbb{P}_{j}^{\dagger},
$$

or, replace the coin and/or the shift operators by non-unitary operators, depending on what sort of decoherence or measurements we are considering.

Most of this section consists of unpacking equation (48) into specific cases and analysing the effects. One may ask, for example, how quickly does the quantum walk become classical as the decoherence is increased: are quantum walks sensitive to small amounts 
of decoherence, or are the quantum effects robust under environmental disturbance. The first studies of decoherence, beyond merely verifying that the classical limit is a classical random walk, were analyses of the likely errors in proposed experimental implementations in Travaglione and Milburn (2002), Sanders et al. (2003) and Dür et al. (2002). These, too, were focused on the properties of the pure quantum walk, and we will provide a short summary in Section 7. The first consideration of the algorithmic properties of partly decohered quantum walks was in Kendon and Tregenna (2003), which used numerical simulation on a variety of discrete coined quantum walks. We will briefly review their results for the walk on the line to provide an overview of the typical effects of decoherence, then examine some of the analytical calculations that confirm and expand these initial observations. An alternative approach is to model the entire system of quantum walker plus environment, and we include examples of this in Section 5.4, where the different methods of decohering the coin are compared.

\subsection{Effects in the walk on the line}

We will begin by unpacking the superoperator notation of equation (48) into the specific case of decoherence events or measurements occurring independently at each time step,

$$
\boldsymbol{\rho}(t+1)=(1-p) \mathbf{S C} \boldsymbol{\rho}(t) \mathbf{C}^{\dagger} \mathbf{S}^{\dagger}+p \sum_{j} \mathbb{P}_{j} \mathbf{S C} \boldsymbol{\rho}(t) \mathbf{C}^{\dagger} \mathbf{S}^{\dagger} \mathbb{P}_{j}^{\dagger} .
$$

Here $\mathbb{P}_{j}$ is a projection that represents the action of the decoherence and $p$ is the probability of a decoherence event happening per time step, or, completely equivalently mathematically, to a weak coupling between the quantum walk system and some Markovian environment with coupling strength $p$. Equation (49) readily lends itself to numerical simulation since $\boldsymbol{\rho}, \mathbf{S}$ and $\mathbf{C}$ can be manipulated as complex matrices, while the $\mathbb{P}_{j}$ generally remove some or all of the off-diagonal entries in $\boldsymbol{\rho}$. Kendon and Tregenna (2003) took equation (49) and evolved it numerically for various choices of $\mathbb{P}_{j}$ : projection onto the position space; projection into the coin space in the preferred basis $| \pm 1\rangle$; and projection of both coin and position. Motivated by the likely form of experimental errors, they also modelled an imperfect Hadamard by applying a Gaussian spread of standard deviation $\sqrt{p} \pi / 4$ about the perfect value of $\pi / 2$ implicit in equation (8): cf. Mackay et al. (2002). Shapira et al. (2003) later modelled, again numerically, imperfect quantum walk operations such as this in more detail and drew the same conclusions, while Konno (2005b) treated the general case of a randomised coin, showing analytically that the classical random walk is obtained. An imperfect shift by the walker has been studied in Dür et al. (2002), see Section 7, and also (in the form of broken links) in Romanelli et al. (2004), see Section 5.3.

To quantify the change in behaviour in the walk on the line, we can calculate the standard deviation, equation (10), which is now also a function of the decoherence rate. In each of these cases, Kendon and Tregenna (2003) found the same general form for the decay of $\sigma(T, p)$ from the quantum to the classical value, with small differences in the rates, as shown in Figure 4. The slope of $\sigma(T, p)$ is finite as $p \rightarrow 0$ and zero at $p=1$. The decay of the spreading rate (as quantified by $\sigma(T, p)$ ), is much as one might have 


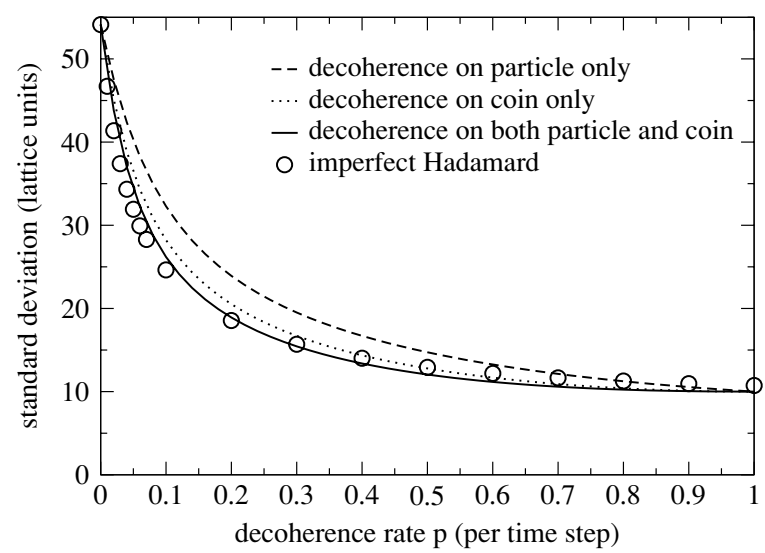

Fig. 4. Standard deviation $\sigma(T, p)$ of the position on a line for different models of decoherence, for $T=100$ time steps.

(Reprinted with permission from Kendon and Tregenna, Phys. Rev. A, 67, 042315 (2003).

Copyright 2003 by the American Physical Society.)

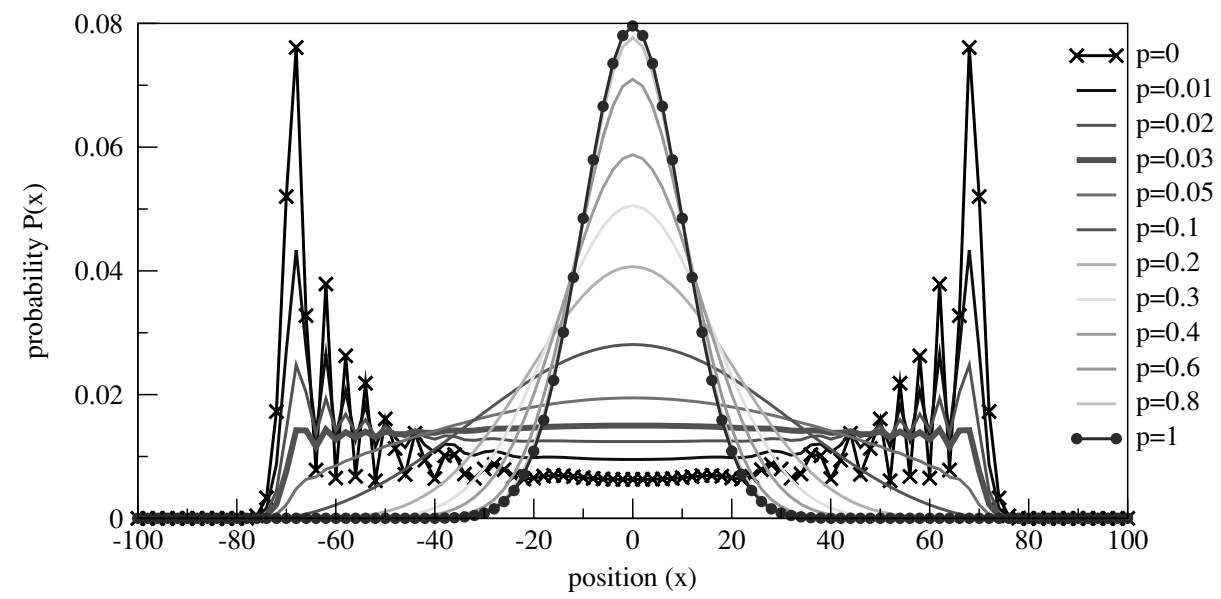

Fig. 5. A quantum walk on a line of 100 steps is progressively decohered by random measurements with probability $p$ per time step as given in the key. For $p=0.03$, an approximately 'top-hat' distribution is obtained.

expected given that quantum states are generally fragile in the face of environmental disturbance. The interesting feature is seen in the shape of the distribution of the position as decoherence begins to take effect. The changing shape as decoherence is increased is shown in Figure 5. Note the good approximation to a top-hat distribution between $\pm T / \sqrt{2}$ that appears for $p=0.03$. For computational physicists who use random walks to sample distributions, this is a very desirable feature, since it provides uniform sampling over a specific range. Moreover, this result is only obtained when decohering the position: compare the three examples in Figure 6. The optimal decoherence rate $p_{u}$ can be obtained by calculating the total variational distance, equation (13), between the actual distribution and an ideal top-hat distribution, The optimum decoherence rate depends on the number 


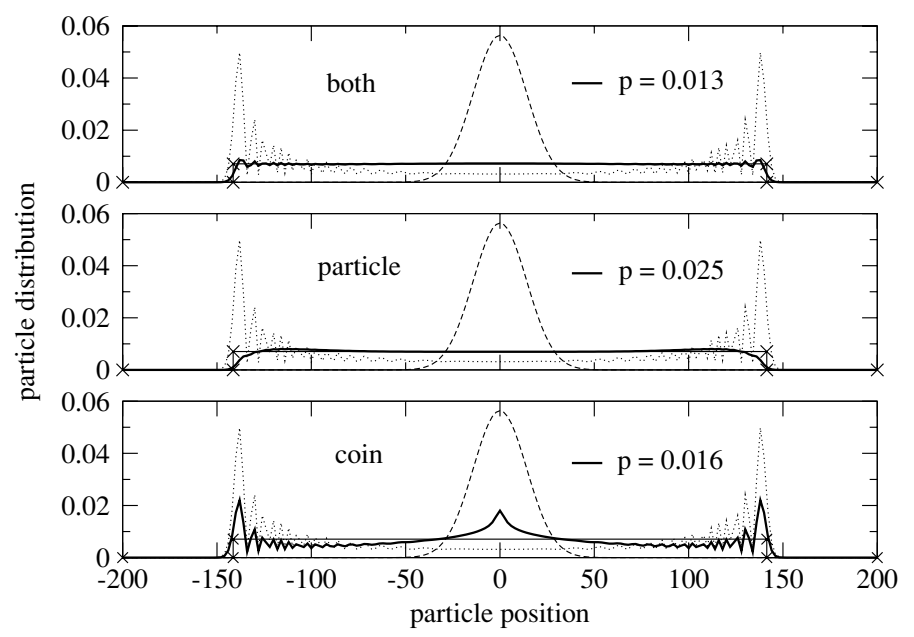

Fig. 6. Distribution of the position for a quantum walk on a line after $T=200$ time steps. Pure quantum (dotted), fully classical (dashed), and decoherence at the rate shown on part of the system indicated by the key (solid). The uniform distribution between $-T / \sqrt{2} \leqslant x \leqslant T / \sqrt{2}$ (crosses) is also shown.

(Reprinted with permission from Kendon and Tregenna, Phys. Rev. A, 67, 042315 (2003).

Copyright 2003 by the American Physical Society.)

of steps in the walk: $p_{u} T \simeq 2.6$ for decoherence on both coin and position and $p_{u} T \simeq 5$ for decoherence on the position only (obtained numerically in Kendon and Tregenna (2003)). Intriguingly, recent work by Maloyer and Kendon shows that the optimal top-hat occurs for the decoherence rate that just removes all the quantum correlations by the end of the quantum walk (Maloyer and Kendon 2007).

Decoherence on the coin only does not produce a top-hat distribution, instead the distribution develops a cusp as it passes from quantum to classical. Lopéz and Paz (2003) provides some insight into why decoherence on the coin and on the position produce qualitatively different results. Lopéz and Paz present an elegant analysis of decoherence in the quantum walk using a discrete Wigner function to bring out the pattern of correlations and their decay when the coin is subject to decoherence. Their decoherence model follows much the same approach as that in Brun et al. (2003a), which will be described in Section 5.2. In their study, Lopéz and Paz restrict the dynamics to cycles. However, most of their results are for small numbers of time steps such that the walk has not joined up round the cycle, and the results are identical to the walk on the line. Wigner functions represent quantum dynamics in a quantum phase space, thus showing the behaviour of both position and momentum degrees of freedom in the same picture. This brings out beautifully the fact that while decohering the coin state is sufficient to reduce a quantum walk on a cycle to a classical walk, if the walker started in a superposition of two position states, this remains untouched by the decoherence. Such a walker with a decohered coin performs a superposition of two classical random walks, each starting at a different position. The converse does not apply, because the position is conditioned on the state of the coin, equation (2), thus decohering the position decohers the coin with it. 
Our first example of decoherence has given us a good guide to what to expect in general: besides the rapid degradation of quantum behaviour, there are interesting effects in particular ranges of low decoherence. These effects are not algorithmically significant, that is, they do not alter the scaling of quantum processing, but, nevertheless, may be useful in practice, and represent intriguing and complex behaviours worthy of study in their own right. For practical purposes (assuming that one day we have a quantum computer available), the improved top-hat profile might still be a useful optimisation to get the most out of the computational resources.

\subsection{Dephasing the coin in the walk on the line}

In the next two sections we will consider the analytical treatment of decoherence in the walk on the line in some detail. First we consider decoherence of the coin only. This will also provide an example of how to use Fourier transforms to simplify the dynamics and obtain the solution. We follow the method of Brun et al. (2003a 2003b) to solve equation (48) for the coined quantum walk on a line with dephasing applied to the coin. We thus have $\mathbf{S}$ given by equation (6),

$$
\mathbf{S}|x, c\rangle=|x+c, c\rangle,
$$

where $|x\rangle$ and $|c\rangle$ are basis states, and $\mathbf{C}$ by equation (8)

$$
\mathbf{C}_{2}^{(\mathrm{H})}=\frac{1}{\sqrt{2}}\left(\begin{array}{rr}
1 & 1 \\
1 & -1
\end{array}\right) .
$$

Transforming from the position basis $|x\rangle$ to the Fourier basis $|k\rangle$ such that

$$
|k, c\rangle=\sum_{x} e^{i k x}|x, c\rangle
$$

we find that

$$
\mathbf{U}_{k}|k, c\rangle=\mathbf{C}_{k}|k, c\rangle
$$

where $\mathbf{C}_{k}$ acts only on the coin degrees of freedom,

$$
\mathbf{C}_{k}=\frac{1}{\sqrt{2}}\left(\begin{array}{rr}
e^{i k} & e^{i k} \\
e^{-i k} & -e^{-i k}
\end{array}\right) .
$$

We now move to density matrix formulation. For a walk starting at the origin in coin state $\left|\psi_{0}\right\rangle$ (not necessarily a basis state),

$$
\boldsymbol{\rho}_{0}=\left|0, \psi_{0}\right\rangle\left\langle 0, \psi_{0}\left|=\int \frac{d k}{2 \pi} \int \frac{d k^{\prime}}{2 \pi}\right| k\right\rangle\left\langle k^{\prime}|\otimes| \psi_{0}\right\rangle\left\langle\psi_{0}\right|,
$$

where $\left|k, \psi_{0}\right\rangle$ is the tensor product $|k\rangle \otimes\left|\psi_{0}\right\rangle$. Using equation (48) to introduce a decoherence operator, we can thus write

$$
\boldsymbol{\rho}(t)=\int \frac{d k}{2 \pi} \int \frac{d k^{\prime}}{2 \pi}|k\rangle\left\langle k^{\prime}\left|\otimes \mathscr{P}_{k k^{\prime}}^{t}\right| \psi_{0}\right\rangle\left\langle\psi_{0}\right|,
$$

where

$$
\mathscr{P}_{k k^{\prime}}\left|\psi_{0}\right\rangle\left\langle\psi_{0}\left|=\sum_{j} \mathbb{P}_{j} \mathbf{C}_{k}\right| \psi_{0}\right\rangle\left\langle\psi_{0}\right| \mathbf{C}_{k^{\prime}}^{\dagger} \mathbb{P}_{j}^{\dagger},
$$


and the set of projectors $\left\{\mathbb{P}_{j}\right\}$ is the decoherence acting on the coin, which we will need to specify in order to carry out an explicit evaluation of equation (54).

While it will in general be difficult to evaluate $\rho(t)$ explicitly, the second moment will give us the main information we need to determine how fast the walk spreads. Calculating the moments allows considerable simplification even before specifying the form of decoherence:

$$
\left\langle x^{m}\right\rangle=\sum_{x} x^{m} P(x, t)=\sum_{x} x^{m} \int \frac{d k}{2 \pi} \int \frac{d k^{\prime}}{2 \pi}\langle x \mid k\rangle\left\langle k^{\prime} \mid x\right\rangle \operatorname{Tr}_{c}\left[\mathscr{P}_{k k^{\prime}}^{t}\left|\psi_{0}\right\rangle\left\langle\psi_{0}\right|\right],
$$

where $T r_{c}[$.] traces over the coin degrees of freedom to aggregate the probability over the different coin states. From equation (50), $\langle x \mid k\rangle=e^{i k x}$, and we may carry out the sum over $x$, giving

$$
\left\langle x^{m}\right\rangle=\frac{(-1)^{m}}{2 \pi} \int d k \int d k^{\prime} \delta^{(m)}\left(k-k^{\prime}\right) \operatorname{Tr}_{c}\left[\mathscr{P}_{k k^{\prime}}^{t}\left|\psi_{0}\right\rangle\left\langle\psi_{0}\right|\right],
$$

where $\delta^{(m)}\left(k-k^{\prime}\right)$ is the $m$ th derivative of the delta function. This can then be integrated by parts.

Brun et al. (2003b) chose pure dephasing for the form of the decoherence on the coin, so the coin projectors in equation (55) are

$$
\mathbb{P}_{ \pm 1}=\frac{1}{\sqrt{2}}\left(\begin{array}{rr}
e^{ \pm i \theta} & 0 \\
0 & e^{\mp i \theta}
\end{array}\right)
$$

This can be solved explicitly, giving in the large $t$ limit

$$
\left\langle x^{2}\right\rangle-\langle x\rangle^{2} \simeq t\left(\cot ^{2} 2 \theta+\csc ^{2} 2 \theta\right)+O(1),
$$

where $\theta$ measures the strength of the dephasing, with $\theta=\pi / 4$ being complete dephasing (classical walk) and $\theta=0$ being a pure quantum walk (the approximation above is not valid for exactly $\theta=0$ ). This shows that even a small amount of decoherence renders the quantum walk classical in the sense that the standard deviation of the position scales as $\sqrt{t}$, but with a larger prefactor the smaller the dephasing: $\cot ^{2} 2 \theta \sim 1 / \theta^{2}$ for small $\theta$.

We will return to decohering the coin in Section 5.4, where a variety of other ways to decohere the coin will be considered and compared. We will now go on to complete the picture for decohering a walk on the line with a calculation of the effects of decoherence in the position.

\subsection{Decohering the position in the walk on the line}

When decoherence acts on the position degrees of freedom, the simplification offered by the factorisation of the dynamics in Fourier space no longer helps: we would have to apply the Fourier transform to the decoherence superoperator as well. The following calculations of the asymptotic behaviour for small $p \ll 1$ illustrate how real space (path counting) methods may be used instead. Following Kendon and Tregenna (2002), we calculate $\sigma(T, p)$ analytically for $p T \ll 1$ and $T \gg 1$ for the case where the $\left\{\mathbb{P}_{j}\right\}$ are the projectors onto the preferred basis $\{|a, x\rangle\}$, that is, decoherence affecting both walker and coin simultaneously. Since, as already explained, decohering the position also decoheres 
the coin, it makes only a small difference whether or not we also decohere the coin explicitly.

The probability distribution for finding the walker in the state $|a, x\rangle$ in the presence of decoherence can be written

$$
P(x, a, T, p)=(1-p)^{T} P(x, a, T)+p(1-p)^{T-1} P^{(1)}(x, a, T)+\ldots,
$$

where $P(x, a, T)$ is the distribution obtained for a perfect walk and $P^{(i)}(x, a, T)$ is the sum of all the ways to have exactly $i$ noise events. For example, we can write

$$
P^{(1)}(x, a, T)=\sum_{t=1}^{T} \sum_{y} \sum_{b} P(y, b, t) P_{y b}(x, a, T-t),
$$

where $P_{y b}(x, a, T-t)$ is the distribution obtained from a perfect walk starting in state $|y, b\rangle$ for $T-t$ steps. For the ideal walk, $\sigma^{2}(T) \equiv \sum_{x} \sum_{a} x^{2} P(x, a, T)$, and for the walk with decoherence,

$$
\sigma^{2}(T, p) \equiv \sum_{x} \sum_{a} x^{2} P(x, a, T, p)
$$

Taking equation (60) to first order in $p$ and substituting along with equation (61) into equation (62) gives to first order in $p T$

$$
\sigma^{2}(T, p) \simeq \sum_{x, a} x^{2}\left\{(1-p T) P(x, a, T)+p \sum_{t=1}^{T} \sum_{y, b} P(y, b, t) P_{y b}(x, a, T-t)\right\} .
$$

The first term on the right-hand side is (by definition) $(1-p T) \sigma^{2}(T)$. Noting that $P_{y b}(x, a, T-t)$ is a translation of a walk starting at the origin, $P_{y b}(x, a, T-t)=P_{0 b}(x-$ $y, a, T-t)$, and relabelling the summed variable $x$ to $(x+y)$ then enables the sums over $x$ and $a$ to be performed in the second term,

$$
\begin{aligned}
p \sum_{t=1}^{T} \sum_{y} \sum_{b} P(y, b, t) & \sum_{x} \sum_{a}(x+y)^{2} P_{0 b}(x, a, T-t) \\
& =p \sum_{t=1}^{T} \sum_{y} \sum_{b} P(y, b, t)\left\{\sigma_{0 b}^{2}(T-t)+2 y\langle x\rangle_{0 b}^{(T-t)}+y^{2}\right\} .
\end{aligned}
$$

From equation (10), $\sigma_{0 b}^{2}(T-t)$ does not depend on $b$, so the summation over $y$ and $b$ may be performed trivially. The remaining summation over $y$ and $b$ applied to $y^{2}$ gives $\sigma^{2}(t)$ by definition. This just leaves the evaluation of

$$
2 p \sum_{t=1}^{T} \sum_{y, b} P(y, b, t) y\langle x\rangle_{0 b}^{(T-t)}=2 p(1-1 / \sqrt{2}) \sum_{t=1}^{T}(T-t) \sum_{y, b} y b P(y, b, t),
$$

where we have used equation (11) for $\langle x\rangle$. We note that this term does not depend on whether the initial coin state is plus or minus one, so we may include both these possibilities equally. Also, by the symmetry of the walk, it is possible to rewrite a probability function for travelling from state $|0, c\rangle$ to $|y, b\rangle$ in the reverse order, that is, as a probability for moving from $|y, b\rangle$ to $|0, c\rangle$. Care must be taken to ensure that the signs of each term due 
to the coefficient $y b$ in the summation are maintained. We obtain

$$
\begin{aligned}
2 p \sum_{t, y, b} P(y, b, t) y\langle x\rangle_{0 b}^{(T-t)} & \\
& =p(1-1 / \sqrt{2}) \sum_{t=1}^{T}(T-t) \sum_{y, b, c}\left(1-2 \delta_{b, c}\right) y b P_{y b}(0, c, t) \\
& \left.=p(1-1 / \sqrt{2}) \sum_{t=1}^{T}(T-t)\left[\sum_{y, b, c} y b P_{y, b}(0, c, t)-2 \sum_{y, b} y b P_{y b}(0, b, t)\right]\right]
\end{aligned}
$$

treating the two parts with and without a delta function independently. Expanding the summations over $b$ and translating the position basis by $-y$ gives

$$
\begin{aligned}
& p \sum_{t=1}^{T} \sum_{y, b} P(y, b, t) y\langle x\rangle_{0 b}^{(T-t)} \\
& \quad=p(1-1 / \sqrt{2}) \sum_{t=1}^{T}(T-t)\left[\sum_{y, c} y P_{0,-1}(y, c, t)-4 \sum_{y} y P_{y,+1}(y,+1, t)\right] .
\end{aligned}
$$

The final summation over $y$ may be bounded above by noting that

$$
\begin{aligned}
P_{0-1}(y,-1, t) & =\left|\left\langle y,-1\left|U^{t}\right| 0,-1\right\rangle\right|^{2} \\
& =\frac{1}{2}\left|\left\langle y+1,1\left|U^{t-1}\right| 0,-1\right\rangle-\left\langle y+1,-1\left|U^{t-1}\right| 0,-1\right\rangle\right|^{2} \\
& \leqslant \frac{1}{2} \sum_{c} P_{0,-1}(y+1, c, t-1) .
\end{aligned}
$$

Using this in equation (67) gives,

$$
\begin{aligned}
2 p \sum_{t, y, b} P(y, b, t) y\langle x\rangle_{0 b}^{(T-t)} & \leqslant p(1-1 / \sqrt{2}) \sum_{t=1}^{T}(T-t)\left[\langle y\rangle_{0,-1}^{t}-2\langle y\rangle_{0,-1}^{(t-1)}+2\right] \\
& \leqslant p(1-1 / \sqrt{2}) \sum_{t=1}^{T}(T-t)[(1-1 / \sqrt{2}) t+\sqrt{2}]
\end{aligned}
$$

where equation (11) has been used for the average values. Note that equation (11) is exact only for asymptotically large times, so the second step in equation (69) introduces further approximations from the contributions to the sum at small times $t$. Combining these results in the full expression for $\sigma^{2}(T, p)$, using equations (63) and (64), and performing the summations over $t$ using $\sum t=T(T+1) / 2$ and $\sum t^{2}=T^{3} / 3+T^{2} / 2+T / 6$ gives

$$
\sigma^{2}(T, p) \leqslant \sigma_{0}^{2}(T)\left[1-\frac{\sqrt{2}}{6} p T+p(\sqrt{2}-1)+\ldots\right] .
$$

Taking the square root gives as an upper bound on the standard deviation,

$$
\sigma(T, p) \leqslant \sigma(T)\left[1-\frac{p T}{6 \sqrt{2}}+\frac{p}{\sqrt{2}}(1-1 / \sqrt{2})+O\left(p^{2}, 1 / T\right)\right] .
$$

Equation (71) compares well with simulation data in Kendon and Tregenna (2003), once a second-order correction for $\sigma(T)=(1-1 / \sqrt{2})^{1 / 2}(T-1 / T)$ is taken into account. 
The bounding procedure applied here is reasonably accurate - numerical studies give the coefficient of $p$ in the above expansion as 0.09566 , compared with the bound of 0.20711 . The first-order dependence is thus proportional to $p T$, the number of decoherence events during the whole quantum walk. For a given decoherence rate $p$, the standard deviation initially decreases linearly in $T$. This calculation first appeared in Kendon and Tregenna (2002).

A similar expansion at the classical end of the full sum in equation (60) gives

$$
P(x, a, T, q)=(1-q)^{T} P^{(T)}(x, a, T)+q(1-q)^{T-1} P^{(T-1)}(x, a, T)+\ldots,
$$

where we have defined $q \equiv(1-p)$ as the small parameter. We know that $P^{(T)}(x, a, T)$ is the classical walk, so

$$
\sigma_{C}^{2}(T)=T=\sum_{x} \sum_{a} x^{2} P^{(T)}(x, a, T)
$$

and we can write

$$
\begin{aligned}
\sigma^{2}(T, q) & =\sum_{x} \sum_{a} x^{2} P(x, a, T, q) \\
& =(1-q)^{T} \sigma_{c}^{2}(T)+q(1-q)^{T-1} \sum_{x} \sum_{a} x^{2} P^{(T-1)}(x, a, T)+\ldots
\end{aligned}
$$

However, to see a difference between the classical and quantum walks, we need to have four consecutive steps of the quantum walk, since the position distributions are identical for the first three steps. The first term that differs from classical is thus derived from part of $P^{(T-4)}(x, a, T)$ given by

$$
P^{(4 c q)}(x, a, T)=\sum_{t=1}^{T-4} \sum_{y, z} \sum_{b, c} P^{(T)}(y, b, t) P_{(y, b)}^{(0)}(z, c, 4) P_{y+z, c}^{(T)}(x, a, T-4-t),
$$

that is, $t$ classical steps, 4 quantum steps, $T-4-t$ classical steps. $\left(P^{(T-4)}(x, a, T)\right.$ also includes combinations with the 4 quantum steps not adjacent to each other.) Since classical segments of the walk are not influenced by the initial state, we may estimate the variance of the total contribution of $P^{(4 c q)}(x, a, T)$ by summing the variances of the segments:

$$
\sigma_{(4 c q)}^{2}(T)=\sum_{t=1}^{T-4}\left[\sigma_{C}^{2}(t)+\sigma^{2}(4)+\sigma_{C}^{2}(T-t-4)\right]=(T-4)\left[T-4+\sigma^{2}(4)\right] .
$$

Explicit calculation $\left(P(x, a, 4)\right.$ has sixteen terms) gives $\sigma^{2}(4)=5$. From the continued expansion of equation (72), the prefactor is $q^{4}(1-q)^{T-4}$, so for small $q$ and large $T$, we have $\sigma^{2}(T, q) \simeq T\left(1+q^{4}\right)$, and for the standard deviation

$$
\sigma(T, q) \simeq \sqrt{T}\left(1+\frac{q^{4}}{2}\right)
$$

to lowest order in $q \equiv 1-p$.

A simpler model of decoherence in both position and coin space by applying measurements at regular intervals and projecting the coin into the $\sigma_{y}$ basis to preserve the symmetry of the walk was solved in Romanelli et al. (2004). They obtain a spreading rate that is basically classical, with a prefactor controlled by the rate of the measurements. 
The average squared distance from the start, measured by the variance, grows as a series of arcs at the quantum rate (quadratic), with regular resetting due to the measurements, pulling the overall rate back to linear (classical). They also generalise to random intervals between measurements, drawing on an analogy with Brownian motion.

Romanelli et al. (2004) also presents a unitary decoherence model affecting the position by analysing a quantum walk in which links between the positions on the line are broken with probability $p$. The transition that should have taken place is turned into a self loop for that step of the walk. The unitary operator for each step is modified to take account of the links that happen to be broken at that time step. For low rates of link-breaking, the quantum behaviour persists, while for high rates the walk makes less progress than even an unimpeded classical random walk.

\subsection{Multiple coins in the walk on the line}

Another analytically tractable approach to reducing coherences in the walk on a line is to enlarge the size of the coin state space, and use parts of it in turn as the walk progresses. This has the advantage of the dynamics remaining purely unitary, rendering the calculations simpler. The first such study, Brun et al. (2003c), considered multiple coins used in sequence, with the sequence repeating after all the coins had been used once. This produces a quantum walk that still spreads linearly with the number of steps, but with the rate of spreading reduced inversely by the number of coins. Only if a new coin is used for every step of the walk does it become equivalent to the classical random walk. This is in contrast with the behaviour obtained by decohering the coin (Brun et al. 2003a), which always results in classical limiting behaviour, as pointed out in Brun et al. (2003b). Classical behaviour is thus associated with an environment so large that one never comes close to the Poincaré recurrence time over the timescales considered.

Related studies that observe or exploit the behaviour when the coin space is limited include Flitney et al. (2004), which uses multiple coins to create a Parrondo game by having the amplitude of the coin flip depend on several previous coins rather than just one. Ermann et al. (2006) uses an enlarged coin space to show explicitly that the behaviour changes from classical back to quantum once the size of the environment space is used up. They use the coins in a random order rather than sequentially as in Brun et al. (2003a). Ribeiro et al. (2004) studied quasi-periodic sequences of coin operators using numerical simulation, and again found different spreading rates, but still an overall linear dependence on the number of steps. More realistic models of a finite-sized environment would have the environment degrees of freedom interacting with each other, but this has not yet been studied in the context of quantum walks.

\subsection{Effects in the walk on the $N$-cycle}

Recall from Section 2.2 that pure discrete-time quantum walks on cycles do not mix (except instantaneously for the few small special cases mentioned in Section 2.3), unless the time-averaged probability distribution, equation (13), is considered. In that case, mixing to the uniform distribution $\bar{M}(\epsilon)$, equation (15), does occur for some choices of 


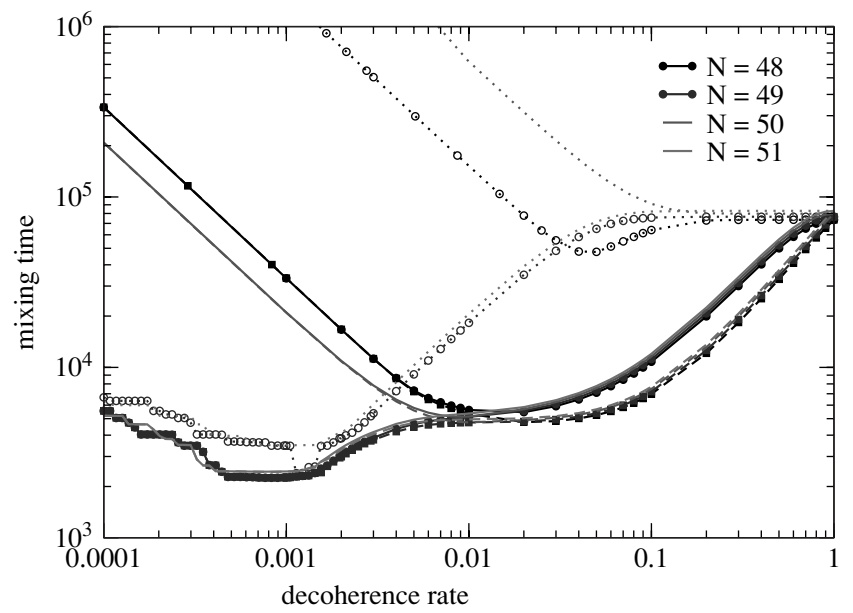

Fig. 7. Numerical data for $\bar{M}_{\epsilon}(p)$ on cycles of size $N=48, N=49, N=50$ and $N=51$ for coin (dotted), position (dashed) and both (solid) subject to decoherence, using $\epsilon=0.002$. Both the axes are logarithmic.

coin operator, but this comes at the cost of requiring a number of steps linear in the inverse accuracy $1 / \epsilon$ instead of logarithmic, unless a number of repetitions are combined in an amplification procedure. Kendon and Tregenna carried out numerical studies of decoherence in cycles (Kendon and Tregenna 2003). They evaluated $\bar{M}(\epsilon, p)$ (the mixing time for $\overline{P(x, T, p)}$, c.f. equation (15)) for walks on cycles of sizes up to $N \simeq 80$, both for pure states and in the presence of the types of decoherence described in Section 5.2 for the walk on a line. They did not include any amplification procedure. For odd- $N$ cycles with no decoherence, they reported that $\bar{M}(\epsilon) \sim N / \epsilon$ as compared to the upper bound of $\bar{M}(\epsilon) \sim N \log N / \epsilon^{3}$ given in Aharonov et al. (2001). Richter has recently confirmed this analytically (Richter 2007b).

With a small amount of decoherence, the mixing time becomes shorter for all cases: typical results are shown in Figure 7. If the coin operator is chosen such that the even- $N$ cycles do not mix to the uniform distribution in the pure quantum walk, the addition of decoherence causes them to mix to the uniform distribution. Although for $N$ divisible by 4 , the coin-decohered mixing time shows a minimum below the classical value at $p \simeq 2 / N$, this mixing time is $\gtrsim N^{2} / 32 \epsilon$, that is, it is still quadratic in $N$. Thus, although noise on the coin causes the even- $N$ cycle to mix to the uniform distribution, it does not produce a significant speed up over the classical random walk. Decoherence on the position produces a minimum mixing time $\bar{M}_{\min }(\epsilon, p)=O(N / \epsilon)$, thus the even- $N$ cycle mixes to uniform in linear time for a suitable choice of decoherence rate $p_{\min } \sim 16 / N^{2}$, independent of $\epsilon$.

For all types of decoherence, the odd- $N$ cycle shows a minimum mixing time at a decoherence rate somewhat earlier than the even- $N$ cycle, roughly $p=2 / N^{2}$, but because of the oscillatory nature of $\overline{P(x, T, p)}$, the exact behaviour is not a smooth function of $p$ or $\epsilon$. (Kendon and Tregenna (2003, Figure 4) illustrates this, and we will show a similar 


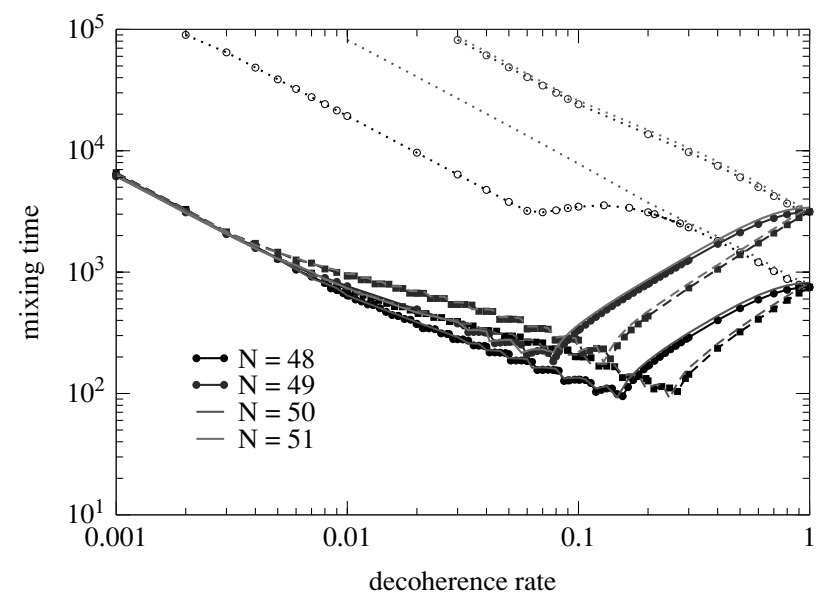

Fig. 8. Numerical data for $M_{\epsilon}(p)$ on cycles of size $N=48$ to $N=51$ for decoherence applied to coin only (dotted), position only (dashed) and both (solid), using $\epsilon=0.002$. Both the axes are logarithmic.

example later.) As decoherence on the position (or both coin and position) increases, the oscillations in $\overline{P(x, T, p)}$ are damped out. At $p \simeq 16 / N^{2}$, the mixing time passes smoothly through an inflexion, and from then on behaves in a quantitatively similar manner to the adjacent-sized even- $N$ cycles, including scaling as $\bar{M}_{\min }(\epsilon, p)=O(N / \epsilon)$ at the inflexion. Thus for $0<p \lesssim 16 / N^{2}$ there is a region where the mixing time stays linear in $N$.

However, one can do better than this: decoherence causes the quantum walk to mix to the uniform distribution without any averaging of $P(x, T, p)$, thus retaining the logarithmic scaling with $\epsilon$ for the mixing times. It is obvious that decoherence must do this, since high decoherence rates reproduce the classical random walk, which has this property. The only question is whether it does so effectively enough to be useful, and the answer is that it does for decoherence on the position (Kendon and Maloyer 2007; Richter $2007 \mathrm{~b})$. Figure 8 shows the mixing time $M(\epsilon, p)$ corresponding to $P(x, T, p)$ as a function of $p$ for cycles of size 48 to 51 . Since even-sized cycles only have support on half the positions at any one time, we have defined the uniform distribution only on the sites where it has support (we can easily fix this if necessary by averaging over two consecutive time steps). It thus behaves like an odd-sized cycle of half the size. Other than this technicality, the behaviour is the same, oscillating peaks end abruptly at the minimum mixing time, followed by a smooth rise to the classical value as the decoherence rate is turned up. The decoherence rate at the minimum mixing time is approximately $\pi / N$ (odd-sized) or $2 \pi / N$ (even-sized), and the mixing time itself scales as $O(N \log (1 / \epsilon))$. This thus provides a quadratic improvement over the classical mixing time, which scales as $O\left(N^{2} \log (1 / \epsilon)\right)$.

If we take a look at the behaviour of $\left\|P(x, t, p)-P_{u}\right\|_{\mathrm{tv}}$, which is shown in Figure 9 for $N=49$, we can see this too is fluctuating at the quantum end, with the period of 


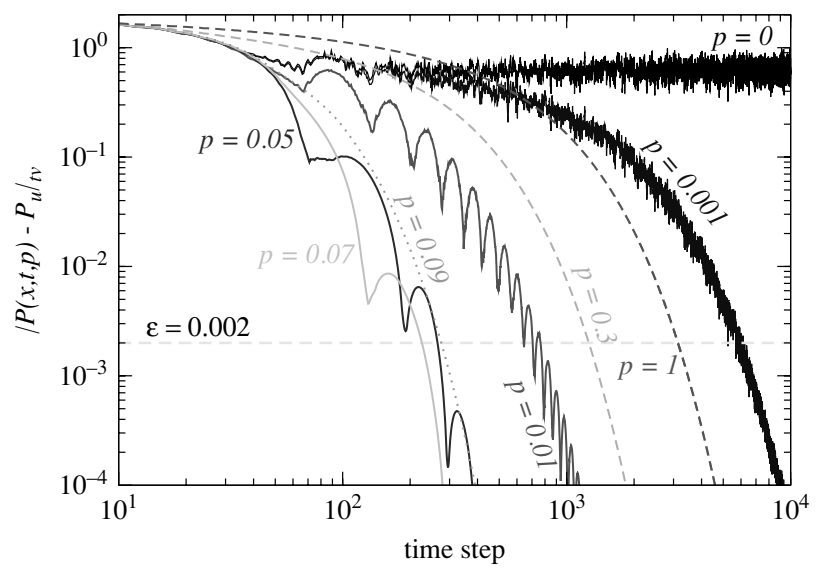

Fig. 9. Difference between the probability distribution $P(x, T, p)$ and the uniform distribution expressed as the total variational distance, equation (13), for $N=49$ for the case with decoherence on both coin and position. Both the axes are logarithmic. The value of $\epsilon$ used in Figures 7 and 8 is shown as a horizontal line.

fluctuation lengthening as it reaches the minimum. On the classical side of the minimum it increases smoothly to the classical value. The time at which the curves last cross the horizontal line at $\epsilon=0.002$ is the mixing time as plotted in Figure 8. In the quantum regime, a different choice of $\epsilon$ thus causes a jump in the value of $M(\epsilon, p)$ if it happens to touch the next peak in $\left\|P(x, t, p)-P_{u}\right\|_{\text {tv }}$. This transition from underdamped (oscillating) to overdamped (smooth) has been observed in a number of decoherence studies, such as the hypercube (Kendon and Tregenna 2003), which is described in the next section, and the studies of decoherence in continuous-time quantum walks described in Section 6. The critical damping point also seems to be associated with the point at which all quantum correlations are destroyed by the decoherence (Maloyer and Kendon 2007), just as this marks the optimal top-hat distribution in the walk on the line.

Richter (2007b) actually proves not only that the optimal mixing time is $O(N \log (1 / \epsilon))$, but also that three different strategies for optimising the mixing time are equally effective. The 'warm start' from Aharonov et al. (2001) can be viewed as running the quantum walk for several segments of random length separated by measurements. The decoherence applied with probability $p$ is a quantum walk of length $T$ interrupted by $p T$ measurements at random times. Simply running the quantum walk for several segments of equal length with measurements applied at the end of each is also effective: the random outcomes from the measurements are sufficient to optimise the mixing. This shows that the effects of decoherence are quite robust, being insensitive to the precise way in which it is applied. The overall conclusion is thus the same as for the walk on a line: there is a useful window within which decoherence enhances rather than degrades the quantum features of the walk. Moreover, it is actually necessary to ensure that the scaling with precision $\epsilon$ is efficient. Additionally, decoherence ensures the quantum walk mixes to a uniform distribution, regardless of the initial conditions or choice of coin operator. 


\subsection{Effects in the walk on the hypercube}

Kempe (2003b; 2005) picked up from where Moore and Russell (2002) left off, and analysed hitting times in discrete-time quantum walks on the hypercube. By the hitting time, we mean the time it takes for the quantum walk to reach the opposite corner from where it started. Kempe considered two types of hitting times, one-shot, where a measurement is made after a predetermined number of steps, and concurrent, where the desired location is monitored continuously to see if the walker has arrived. While the one-shot hitting time is a pure quantum dynamics, the continuous monitoring of the concurrent hitting time measurement removes part of the quantum coherences, thus it constitutes a type of decoherence. More recently, Krovi and Brun (2006a) defined a hitting time in closer analogy with the usual classical hitting time: the average time of first arrival at the target location.

Following Kempe, the one-shot hitting time $H_{\text {one-shot }}\left(r_{0}\right)$ between $\left|x_{0}\right\rangle$ and $\left|x_{f}\right\rangle$ is

$$
H_{\text {one-shot }}\left(r_{0}\right)=\left\{T:\left|\left\langle x_{f}\left|\mathbf{U}^{T}\right| x_{0}\right\rangle\right|^{2} \geqslant r_{0}\right\},
$$

where $r_{0}$ is a threshold probability of being at the target state, $\mathbf{U}$ is the evolution of one step of the walk, $\left|x_{0}\right\rangle$ is the starting state and $\left|x_{f}\right\rangle$ is the location being hit.

To define the remaining two hitting times, we need to define a measured quantum walk - Kempe, and Krovi and Brun give essentially equivalent definitions. For hitting a single final location, we need a measurement with two outcomes (this is easily generalised), say $\mathbb{P}_{f}$ and $\mathbb{Q}_{f}=\mathbb{1}-\mathbb{P}_{f}$, where $\mathbb{P}_{f}=\left|x_{f}\right\rangle\left\langle x_{f}\right| \otimes \mathbb{1}_{c}$, that is, the projector onto the state $\left|x_{f}\right\rangle$ for any coin state. If the walker is found at $\left|x_{f}\right\rangle$, the walk is assumed to have ended, it acts as an absorbing boundary. If the walk does not reach $\left|x_{f}\right\rangle$ after $t$ steps, the state of the walk can be written

$$
\boldsymbol{\rho}(t)=\frac{\mathscr{U}_{Q}^{t} \boldsymbol{\rho}}{\operatorname{Tr}\left[\mathscr{U}_{Q}^{t} \boldsymbol{\rho}\right]}
$$

where $\mathscr{U}_{Q} \boldsymbol{\rho}=\mathbb{Q}_{f} \mathbf{U} \boldsymbol{\rho} \mathbf{U}^{\dagger} \mathbb{Q}_{f}^{\dagger}$. This differs from equation (48) in that we keep only one outcome from the measurement and renormalise the density matrix. Since we know the measurement outcome, we know whether the walk has arrived or not, so we only need to keep the part that has not yet arrived at $\left|x_{f}\right\rangle$. The first arrival (which is also called the first crossing) probability after $t$ steps can thus be written

$$
r(t)=\operatorname{Tr}\left[\mathscr{U}_{P} \mathscr{U}_{Q}^{t-1} \boldsymbol{\rho}\right] .
$$

Kempe defines the concurrent hitting time as

$$
H_{\text {concurrent }}\left(r_{0}\right)=\min \left\{T \mid \exists t<T: r(t)>r_{0}\right\} .
$$

The hitting time defined by Krovi and Brun is

$$
H_{\text {average }}=\sum_{t=0}^{t=\infty} t r(t),
$$

which is the average arrival time. Krovi and Brun compare the concurrent hitting time with their average hitting time and find they both scale in a similar polynomial manner, compared with the exponentially long hitting time of a classical random walk, provided 


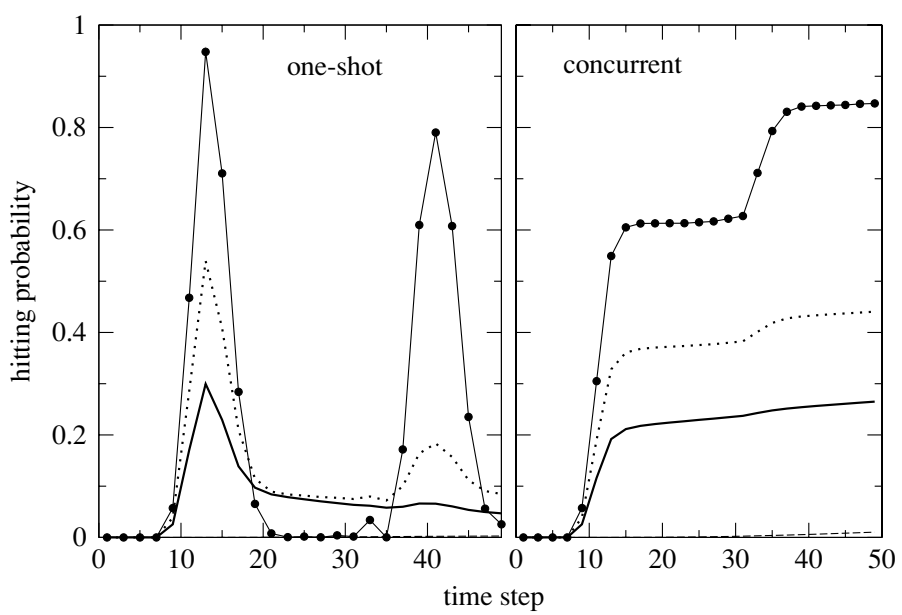

Fig. 10. Hitting probability on a 9-dimensional hypercube for one-shot (left) and concurrent (right), perfect walk (circles), with $p=0.05$ (dotted), $p=0.1 \simeq 1 / 9$ (solid). The classical hitting probability is barely visible (dashed).

(Reprinted with permission from Kendon and Tregenna, Phys. Rev. A, 67, 042315 (2003).

Copyright 2003 by the American Physical Society.)

they use a Grover coin for the quantum walk, and consider $\left|x_{0}\right\rangle=|0 \ldots 0\rangle$ and $\left|x_{f}\right\rangle=$ $|1 \ldots 1\rangle$. Their numerical results suggest the bound of $H_{\text {concurrent }}(\Omega(1)) \leqslant O\left(n^{2} \log n^{2}\right)$ obtained by Kempe $(2003 \mathrm{~b} ; 2005)$ is not tight, the actual hitting time seems to be better than this in the large- $n$ limit.

Krovi and Brun also investigated the effects of breaking the symmetry of the Grover coin by using a DFT coin and by distorting the hypercube lattice. The DFT coin has the property that, for certain configurations, the hitting time becomes infinite, while a Grover coin with a small distortion of the hypercube lattice increases the hitting time somewhat, though it is still less than the classical random walk hitting time. Analogous results for coined quantum walks on the 'glued trees' graph were noted in Tregenna et al. (2003). Further work by Krovi and Brun on the question of whether and under what conditions quantum walks show dramatically different properties (speed up or slow down) compared with classical random walks suggests that it is highly dependent on the symmetry of the graph (Krovi and Brun 2006b). For the Grover coin, this is exemplified by the quantum walk search algorithm of Shenvi et al. (2003) (which is described in Section 4.1), where any disturbance of the symmetry causes the walker to converge on the marked state.

Kendon and Tregenna (2003) investigated the effects of adding extra decoherence to the quantum walk on a hypercube over and above that implied by the measurements of the target location. For both one-shot and concurrent hitting times (this work predates Krovi and Brun (2006a)), the key parameter is the probability $r(t)$ of finding the walker at the chosen location. Their numerical calculations show that all forms of decoherence have a similar effect on $r(t)$, see Figure 10, reducing the peaks and smoothing out the troughs. This is useful for the one-shot hitting time, raising $r(t)$ in the trough to well above the classical value, so it is no longer necessary to know exactly when to measure. 
For $p \lesssim 1 / n$, the height of the first peak scales as $r_{p}(t)=r(t)(0) \exp \{-(n+\alpha) p\}$, where $0 \lesssim \alpha \lesssim 2$, depending on whether the coin, the position or both are subject to decoherence. An exponential decrease in the presence of decoherence sounds about as bad as it could reasonably be, and for long times, of course, decoherence reduces the walk to classical behaviour. However, the hitting times are short, only $\sim n \pi / 2$ steps, so $p \simeq 1 / n$ only lowers $r(t)$ by a factor of $1 / e$. This is insignificant for algorithmic purposes, as it is only a factor of order unity and thus still exponentially better than classical. Note also that the size of the graph (measured in number of nodes) is $2^{n}$, so the decoherence only has a linear effect measured in terms of the size of the graph.

The concurrent hitting time already includes a portion of decoherence (no extra features are produced by the addition of unselective decoherence), but there is still a range of $0<p \lesssim 1 / n$ within which the quantum speed up is preserved. Note that in both the one-shot and concurrent cases, $p \simeq 1 / n$ is a critical damping rate, smoothing out the second peak (shown at around $40(\equiv 3 n \pi / 2)$ steps in Figure 10).

Decoherence in discrete-time walks has thus provided us with a number of notable common features, such as smoother spreading, enhanced mixing, and a transition from under-damped (quantum) to over-damped (classical) behaviour, which is analogous to classical damping in a harmonic oscillator. We will see in the next section that these features also appear for the continuous-time quantum walk under decoherence.

\section{Decoherence in continuous-time walks}

Less work has been done on decoherence in continuous-time quantum walks, probably in part because the numerical simulations require more resources (to integrate rather than iterate the dynamics). Analytical calculations for the continuous-time walk are often simpler than for the discrete-time walk due to the lack of a coin, but in the case of decoherence this removes the simple case of decoherence on the coin only that has been so successfully studied in the discrete-time walk. Nonetheless, there have been two notable recent analytical results. Fedichkin et al. (2006) gives the scaling for mixing times for the walk on the cycle for both small and large decoherence rates. They augment this with numerical studies showing a minimum mixing time for intermediate decoherence rates. Alagic and Russell (2005) gives a solution for the decoherent walk on the hypercube for all rates of decoherence. These results will be discussed in separate sections below, but we will first set up a general model for decoherence in continuous-time quantum walks analogous to the basic model used for discrete-time walks. Then we will briefly examine the effects of decoherence in the continuous-time quantum walk on the line, obtained numerically, to illustrate the key features we can expect to see.

The pure state evolution of the continuous-time quantum walk given by equation (28) can be expressed in density matrix form as

$$
\frac{d \boldsymbol{\rho}(t)}{d t}=-i \gamma[\mathbf{A}, \boldsymbol{\rho}],
$$

where $[a, b] \equiv a b-b a$ denotes the commutator. We can add a non-unitary decoherence to this in exact analogy to the discrete-time method by writing

$$
\boldsymbol{\rho}(t+\delta t)=(1-p \delta t)(\boldsymbol{\rho}(t)-i \gamma \delta t[\mathbf{A}, \boldsymbol{\rho}])+p \delta t \mathscr{P} \boldsymbol{\rho}
$$




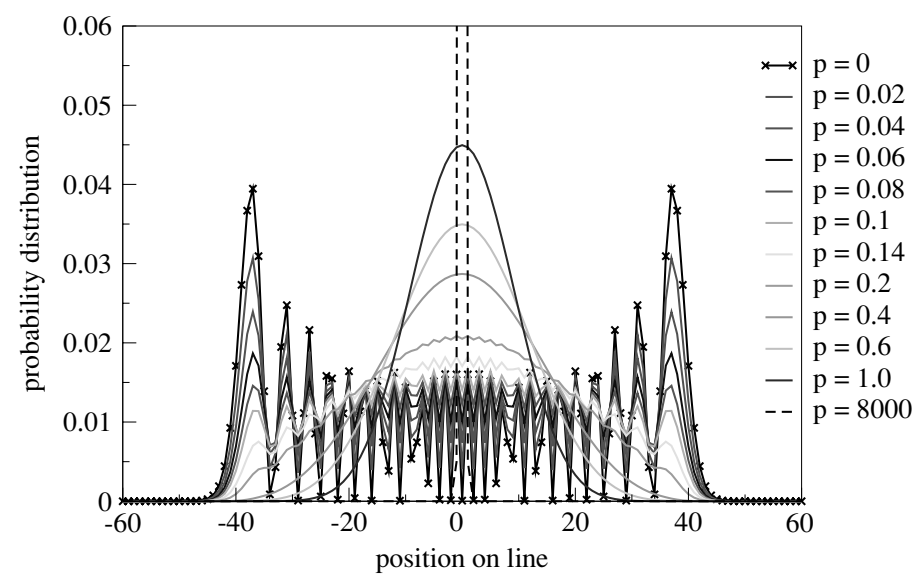

Fig. 11. Probability distribution for a continuous-time quantum walk on the line with $\gamma=0.5$, at time $t=40$, for various decoherence rates $p$ give in the key.

where $\mathscr{P}$ represents the action of the noise: for example, a set of projectors $\left\{\mathbb{P}_{j}\right\}$, such that $\mathscr{P} \boldsymbol{\rho}=\sum_{j} \mathbb{P}_{j} \rho \mathbb{P}_{j}^{\dagger}$. For uncorrelated noise, taking $\delta t \rightarrow 0$, this yields

$$
\frac{d \boldsymbol{\rho}(t)}{d t}=-i \gamma[\mathbf{A}, \boldsymbol{\rho}]-p \boldsymbol{\rho}+p \mathscr{P} \boldsymbol{\rho} .
$$

The effect of the extra two terms is to reduce some or all of the off-diagonal elements of $\boldsymbol{\rho}(t)$ at a rate $p$ per unit time.

\subsection{Effects in the walk on the line}

In contrast to the extensive studies of the discrete-time walk on the line, there are no published analytical treatments of decoherence in a continuous-time quantum walk on the line. We can easily investigate this numerically: for uncorrelated noise events at a rate $p$ per unit time, we can unpack equation (85) into

$$
\frac{d}{d t} \rho_{x, y}(t)=i\left[\frac{\rho_{x, y+1}-\rho_{x+1, y}-\rho_{x-1, y}+\rho_{x, y-1}}{4}\right]-p\left(1-\delta_{x, y}\right) \rho_{x, y},
$$

where $x, y \in \mathbb{Z}$. (Fedichkin et al. (2006) credits this model as having been developed by Gurvitz (1997) and Gurvitz et al. (2003).) Figure 11 shows the results of evolving a continuous-time quantum walk on the line with hopping rate $\gamma=0.5$ until $t=40$ for various decoherence rates. This is to be compared with Figure 5, which shows the same type of study for a discrete-time quantum walk. The continuous-time walk does not produce quite such a nice top-hat as the discrete-time walk, partly due to having support on all sites rather than alternate sites, though it would probably be equally useful in practice. There is, however, a striking new effect: unlike with the discrete-time walk, where decoherence rates larger than one have no useful interpretation, there is no reason why $p$ in equations (85) and (86) cannot be made arbitrarily large. In Figure 11, $p=8000$ is shown: the walk hardly manages to leave the starting point. High values of $p$ can be 
interpreted as continuous monitoring, which projects the walk into the initial state with high probability. In other words, the walk suffers a quantum Zeno effect (Misra and Sudarshan 1977), a phenomenon that does not appear in coined quantum walks. One consequence of this is that quantities such as the concurrent hitting time are infinite, since the continuous monitoring will ensure the continuous-time quantum walk never arrives.

\subsection{Effects in the walk on the $N$-cycle}

The time-dependent non-unitary evolution of $\boldsymbol{\rho}(t)$ on a cycle is also given by equation (86), but with $x, y \in \mathbb{Z}^{N}$ for a cycle of size $N$. Fedichkin et al. (2006) showed how to solve this for small and large values of decoherence. We will now outline their method. The substitution

$$
R_{x, y}=i^{y-x} \rho_{x, y}
$$

converts the differential equations into a set with real coefficients:

$$
\frac{d}{d t} R_{x, y}=\frac{1}{4}\left(R_{x, y+1}+R_{x+1, y}-R_{x-1, y}-R_{x, y-1}\right)-p\left(1-\delta_{x, y}\right) R_{x, y} .
$$

Note that this leaves the diagonal elements of $\rho$ identical to the diagonal elements of $\boldsymbol{R}$. As a side note, Fedichkin et al. observed from these equations that if $p=0$, there is an exact mapping of the quantum walk on a cycle onto a classical random walk on a two-dimensional torus, and if $p \neq 0$, there is still an exact mapping of the quantum walk on a cycle onto some classical dynamics on a directed toric graph. Similar mappings (one-dimensional quantum to two-dimensional classical) also occur in quantum phase transitions in spin chains (see, for example, Sachdev (1999)), and can prove a useful technique for analysing quantum systems, though we will not need to use it explicitly here.

Equation (88) can be expressed as the linear operator equation

$$
\frac{d}{d t} \boldsymbol{R}(t)=(\mathbb{L}+p \mathbb{U}) \boldsymbol{R}(t) .
$$

One can think of $\mathbb{L}$ and $\mathbb{U}$ as $N^{2} \times N^{2}$ matrices and $\boldsymbol{R}(t)$ as a vector of size $N^{2}$ (instead of a matrix of size $N \times N)$. The indices of $\boldsymbol{R}(t)$ as a vector become $x N+y$ where $x, y$ are the pair of indices of $\boldsymbol{R}(t)$ as a matrix, but we will write $x y$ for such indices to reduce the complexity of the notation. The formal solution of (89) can be written $\boldsymbol{R}(t)=e^{t(\mathbb{L}+p \mathbb{U})} \boldsymbol{R}(0)$. The explicit solution for arbitrary decoherence rates has not been obtained, but one can examine the behaviour of the mixing time under the action of small and large decoherence using standard perturbation theory methods. When the decoherence rate $p$ is small such that $p N \ll 1, p \mathbb{U}$ is treated as a perturbation of $\mathbb{L}$. The unperturbed linear operator $\mathbb{L}$ (which evolves the pure state quantum walk in density matrix formalism) has eigenvalues

$$
\lambda_{m n}=i \sin \left(\frac{\pi(m+n)}{N}\right) \cos \left(\frac{\pi(m-n)}{N}\right)
$$

with corresponding eigenvectors

$$
V_{\mu v, m n}=\frac{1}{N} \exp \left(\frac{2 \pi i}{N}(m \mu+n v)\right) .
$$


Here $\mu \nu, m n$ are indices $0 \leqslant \mu, v, m, n<N$ combined like $x, y$ above. With due consideration of the degeneracies in the eigenvalues $\lambda_{m n}$ of $\mathbb{L}$ (see Fedichkin et al. (2006) for details), the eigenvalue perturbation $\tilde{\lambda}_{m n}$ turns out to be

$$
\tilde{\lambda}_{m n}=-p \frac{(N-2)}{N} \text {. }
$$

Thus, the solution is of the form

$$
R_{x y}(t)=\frac{\delta_{x y}}{N}+\frac{1}{N^{2}} \sum_{m n}\left(1-\delta_{[m+n](\bmod N), 0}\right) e^{t\left(\lambda_{m n}+\tilde{\lambda}_{m n}\right)} \exp \left[\frac{2 \pi i}{N}(m x+n y)\right] .
$$

The probability distribution of the continuous-time quantum walk is given by the diagonal terms (considering $\boldsymbol{R}(t)$ as an $N \times N$ matrix again) $P(x, t, p)=R_{x, x}(t)$, that is

$$
\begin{aligned}
& P(x, t, p) \\
& =\frac{1}{N} \\
& +\frac{1}{N^{2}} \sum_{(m, n)}\left(1-\delta_{[m+n](\bmod N), 0}\right) \times\left[\delta_{m, n} e^{-p \frac{N-1}{N} t}+\left(1-\delta_{m, n}\right) e^{-p \frac{N-2}{N} t}\right] \\
& \times \exp \left[i t \sin \left(\frac{\pi(m+n)}{N}\right) \cos \left(\frac{\pi(m-n)}{N}\right)\right] \exp \left[\frac{2 \pi i}{N}(m+n) x\right] .
\end{aligned}
$$

Fedichkin et al. then calculate an upper bound on the mixing time $M(\epsilon)$. Defining

$$
\mathscr{M}_{x}(t)=\frac{1}{N} \sum_{m=0}^{N-1} e^{i t \sin (2 \pi m / N)} \omega_{N}^{m x}
$$

where $\omega_{N}=\exp (2 \pi i / N)$, and noting that

$$
\begin{aligned}
\mathscr{M}_{x}^{2}(t / 2) & =\frac{1}{N^{2}} \sum_{m, n=0}^{N-1} e^{i t \lambda_{(m, n)}} \omega_{N}^{(m+n) x} \\
\mathscr{M}_{2 x}(t) & =\frac{1}{N} \sum_{m=0}^{N-1} e^{i t \lambda_{(m, m)}} \omega_{N}^{2 m x}
\end{aligned}
$$

and that $\left|\mathscr{M}_{x}(t)\right| \leqslant 1$, we can simplify equation (94) as follows,

$$
\begin{aligned}
\left|P(x, t, p)-\frac{1}{N}\right| & \leqslant e^{-p \frac{N-2}{N} t}\left|\mathscr{M}_{x}^{2}(t / 2)+\frac{e^{-t p / N}-1}{N}\left[\mathscr{M}_{2 x}(t)-\frac{2-(N \bmod 2)}{N}\right]\right| \\
& \leqslant e^{-p \frac{N-2}{N} t}\left|1+\frac{e^{-t p / N}-1}{N}(1-2 / N)\right| .
\end{aligned}
$$

Adding a now-trivial summation over $x$ gives us the total variational distance required to obtain the mixing time:

$$
\sum_{x=0}^{N-1}\left|P(x, t, p)-\frac{1}{N}\right| \leqslant e^{-p \frac{N-2}{N} t}\left(N+e^{-t p / N}-1\right) .
$$


Since $e^{-t p / N} \leqslant 1$, the above equation shows that the mixing condition is $N e^{-p \frac{N-2}{N} t} \leqslant \epsilon$. This gives the mixing time bound of

$$
M(\epsilon)<\frac{1}{p} \log \left(\frac{N}{\epsilon}\right)\left[1+\frac{2}{N-2}\right] .
$$

Some comments on this result are in order. First, this is the mixing time for the instantaneous probability distribution, so it should be compared with the discrete-time results in Figure 8. Just as with the discrete-time walk, the first key effect of decoherence is to cause the continuous-time quantum walk to mix. On the face of it, $M(\epsilon)$ appears to scale as $\log (N)$, which would imply an exponential speed up over classical mixing times of $O\left(N^{2}\right)$. However, this formula is only valid for $p N \ll 1$, so the $1 / p$ scaling ensures that $M(\epsilon) \gg N$ for the range of validity of the result. What this result tells us is that decoherence causes the continuous-time quantum walk to mix, with the dominant effect a scaling of $1 / p$, and only weak (logarithmic) dependence on $N$ and $\epsilon$. It does not tell us anything about the optimal decoherence rate that might give a minimum mixing time, nor what value that minimum mixing time might take.

We can calculate the time-averaged mixing time from the results for $P(x, t, p)$ above, and compare it with the discrete-time and classical values. Again, we will obtain a result that is only valid in restricted ranges of parameters. Working with equation (97), and assuming $N \gg 1$ for simplicity, we obtain

$$
\left|\overline{P(x, T, p)}-\frac{1}{N}\right| \leqslant\left|\frac{1}{T} \int_{0}^{T}\left(e^{-p t}+\frac{1}{N}\right) d t-\frac{1}{N}\right| \leqslant\left|\frac{1}{p T}\left(1-e^{-p T}\right)\right| .
$$

Summing over $x$ to obtain the total variational distance trivially multiplies by $N$. Comparing this to $\epsilon$ as before gives

$$
\frac{N}{p T}\left(1-e^{-p T}\right) \leqslant \epsilon
$$

Assuming $p T \gg 1$ (which is consistent with our other assumptions, $N \gg 1$ and $p N \ll 1$, it requires $T \gg N$ ) gives

$$
\bar{M}(\epsilon) \geqslant \frac{N}{p \epsilon},
$$

which is similar to the scaling found numerically for the discrete-time quantum walk. In particular, the scaling with $N$ and $\epsilon$ is no longer logarithmic, as a consequence of the time-averaging. The scaling with $p$ is still inverse, indicating that, in general, pure continuous-time quantum walks on cycles do not mix to within $\epsilon$ of uniform, even when time-averaged distributions are considered, as already noted in Section 3.3.

We now turn to the analogous calculation for large decoherence rates. When the decoherence rate $p$ is large, that is, when $p \gg 1$, the quantum walk experiences a quantum Zeno effect that suppresses most of the transitions. We can therefore neglect all but the first off-diagonal terms in the matrix $\boldsymbol{R}(t)$ from equation (88). Retaining only matrix elements that are of order $1 / p$ produces a truncated set of differential equations for the 
elements along the major and the two adjacent minor diagonals:

$$
\begin{aligned}
R_{x, x}^{\prime} & =\frac{1}{4}\left(R_{x, x+1}+R_{x+1, x}-R_{x-1, x}-R_{x, x-1}\right) \\
R_{x, x+1}^{\prime} & =\frac{1}{4}\left(R_{x+1, x+1}-R_{x, x}\right)-p R_{x, x+1} \\
R_{x, x-1}^{\prime} & =\frac{1}{4}\left(R_{x, x}-R_{x-1, x-1}\right)-p R_{x, x-1} .
\end{aligned}
$$

To facilitate our subsequent analysis, we define

$$
\begin{aligned}
& a_{x}=R_{x, x} \\
& d_{x}=R_{x, x+1}+R_{x+1, x} .
\end{aligned}
$$

Then, we observe that

$$
\begin{aligned}
& a_{x}^{\prime}=\frac{\left(d_{x}-d_{x-1}\right)}{4} \\
& d_{x}^{\prime}=\frac{\left(a_{x+1}-a_{x}\right)}{2}-p d_{x} .
\end{aligned}
$$

The solution of the differential equation at large $p$ has the form

$$
a_{x}=\frac{1}{N} \sum_{y=0}^{N-1} \exp \left(-\frac{\sin ^{2} \frac{\pi y}{N}}{2 p} t\right) \omega^{x y} .
$$

Based on the above analysis, the full solution for $R(t)$ is given by

$$
R_{x, y}(t)= \begin{cases}a_{x} & \text { if } x=y \\ d_{x} / 2 & \text { if }|x-y|=1 \\ 0 & \text { otherwise. }\end{cases}
$$

The total variation distance between the uniform distribution and the probability distribution of the decoherent quantum walk is given by

$$
\sum_{x=0}^{N-1}\left|a_{x}(t)-\frac{1}{N}\right|=\sum_{x=0}^{N-1}\left|\frac{1}{N} \sum_{y=0}^{N-1} \exp \left(-\frac{\sin ^{2} \frac{\pi y}{N}}{2 p} t\right) \exp \left(\frac{2 \pi i x y}{N}\right)-\frac{1}{N}\right|,
$$

which simplifies to

$$
\sum_{x=0}^{N-1}\left|a_{x}(t)-\frac{1}{N}\right|=\frac{1}{N} \sum_{x=0}^{N-1}\left|\sum_{y=1}^{N-1} \exp \left(-\frac{\sin ^{2} \frac{\pi y}{N}}{2 p} t\right) \cos \left(\frac{2 \pi y x}{N}\right)\right| .
$$

A lower bound on the mixing time for large decoherence rate $p$ can be derived as follows. Note that

$$
\begin{aligned}
\sum_{x=0}^{N-1}\left|a_{x}(t)-\frac{1}{N}\right| & \geqslant\left|a_{0}(t)-\frac{1}{N}\right|=\frac{1}{N} \sum_{y=1}^{N-1} \exp \left(-\frac{\sin ^{2} \frac{\pi y}{N}}{2 p} t\right) \\
& \geqslant \frac{2}{N} \exp \left(-\frac{\sin ^{2} \frac{\pi}{N}}{2 p} t\right),
\end{aligned}
$$




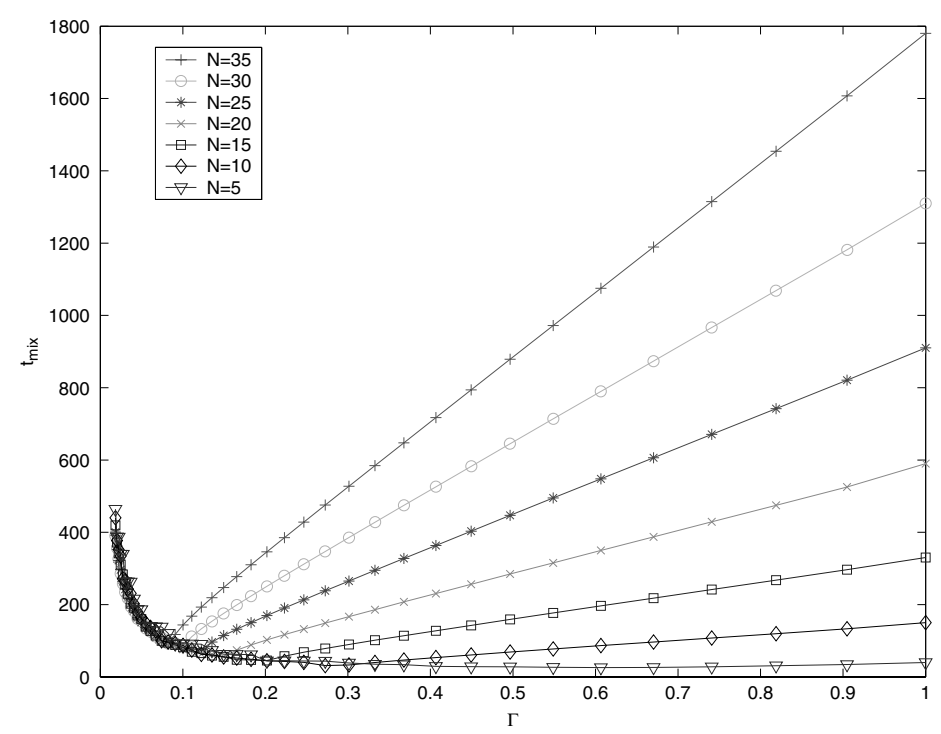

Fig. 12. The quantum to classical transition of mixing time in a continuous-time decoherent quantum walk on $C_{N}$ for $N=5,10,15,20,25,30,35$.

(From Fedichkin et al. (2006); reproduced with permission of Rinton Press.)

where the first inequality uses the term $x=0$ only and the second inequality uses the terms $y=1, N-1$. This expression is monotone in $t$, and is a lower bound on the total variation distance. It reaches $\epsilon$ at time $T_{\text {lower }}$, when

$$
T_{\text {lower }}=\frac{2 p}{\sin ^{2} \frac{\pi}{N}} \ln \left(\frac{2}{N \epsilon}\right) \simeq \frac{2 p N^{2}}{\pi^{2}} \ln \left(\frac{2}{N \epsilon}\right)
$$

for large $N \gg 1$.

An upper bound on the mixing time for large decoherence rate $p$ can also be derived (see Fedichkin et al. (2006) for details)

$$
T_{\text {upper }}=\frac{p N^{2}}{2} \ln \left(\frac{2+\epsilon}{\epsilon}\right) .
$$

For large decoherence rates $p \gg 1$, the mixing times are bounded as

$$
\frac{p N^{2}}{\pi^{2}} \ln \left(\frac{2}{N \epsilon}\right)<M(\epsilon)<\frac{p N^{2}}{2} \ln \left(\frac{2+\epsilon}{\epsilon}\right) .
$$

These bounds show that $M(\epsilon)$ is linearly proportional to the decoherence rate $p$, but is quadratically dependent on $N$, and logarithmically dependent on $\epsilon$.

The change in the form of the scaling with $p$ from reciprocal for small $p$ to linear for large $p$ suggests there is a minimum mixing time at some intermediate decoherence rate. Fedichkin et al. (2006) confirmed this numerically: see Figure 12. 
The effects of decoherence on continuous-time quantum walks on cycles is thus similar to the effects on discrete-time walks on cycles, as described in Section 5.5. In fact, the proofs in Richter (2007b) are sufficiently general that they apply equally to the continuous-time walk as to the discrete-time walk. This fills the gap between small and large decoherence rates, and proves that continuous-time walks, with $O(\log (1 / \epsilon))$ decoherence or measurement events, mix in time $O(N \log (1 / \epsilon))$ on the cycle (and the $d$-dimensional torus).

\subsection{Effects in the walk on the hypercube}

The hypercube is an attractive subject for analytical calculations of quantum walks because, under certain conditions, it offers two routes to simplify the analysis. If the walk has the appropriate symmetry, it is constrained to a subspace that maps to a walk on a line segment with positions corresponding to the Hamming weight of the node labels (with position dependent transition rates). Alternatively, the continuous-time walk can be factored in Fourier space into independent walks on each qubit of the labels of the locations, as explained in Section 3.4. Furthermore, both mixing properties for spreading over the whole graph, and hitting properties for traversing to opposite corners are non-trivial on the hypercube. We thus have a large number of comparisons to make between discrete and continuous-time quantum walks, and classical random walks. Alagić and Russell (2005) provides a complete solution to the dynamics of the continuous-time quantum walk on the hypercube subject to decoherence. We will sketch their method of solution, then discuss their results.

Alagic and Russell give their quantum walk an energy $k$ - this is equivalent to an arbitrary hopping rate $\gamma=k / n$ instead of the choice of $\gamma=1 / n$ for a graph with vertices of degree $n$. We consider the continuous quantum walk on the $n$-dimensional hypercube with energy $k$ and decoherence rate $p$, starting from the initial wave function $\Psi_{0}=|0\rangle^{\otimes n}$, corresponding to the corner with Hamming weight zero. The decoherence operators in equation (85) project the walker onto one of the vertices of the hypercube chosen uniformly at random. It is useful to write these projectors explicitly in terms of single qubit projectors $\Pi_{0}$ and $\Pi_{1}$ onto $|0\rangle$ and $|1\rangle$, respectively. We have

$$
\mathbb{P}=\frac{1}{n} \sum_{1 \leqslant j \leqslant n}\left[\Pi_{0}^{j} \otimes \Pi_{0}^{j}+\Pi_{1}^{j} \otimes \Pi_{1}^{j}\right]
$$

where $\Pi_{0}^{j}=\mathbb{1} \otimes \cdots \otimes \mathbb{1} \otimes \Pi_{0} \otimes \mathbb{1} \otimes \cdots \otimes \mathbb{1}$ with the non-identity projector appearing in the $j$ th place, and similarly for $\Pi_{1}^{j}$.

We now show that with the model of decoherence described above each dimension still behaves independently. Recall equation (41) showing that the adjacency matrix for the hypercube decomposes into a sum of tensor products, with each acting only on a single qubit. Since the noise operators $\mathbb{P}_{j}$ also have this structure, provided $\rho(0)$ starts off in a state that is also decomposable (such as the all-zero vertex), the subsequent evolution will maintain this structure and will remain decomposable as a system of $n$ non-interacting qubits. Since the qubits are now in mixed states $\boldsymbol{\rho}_{j}$, the superoperator acting on them is 
equivalent to a $4 \times 4$ matrix:

$$
\begin{aligned}
\mathscr{J} & =\frac{t}{n}\left[\left(\mathbb{1} \otimes i n \sigma_{x}\right)-\left(i n \sigma_{x} \otimes \mathbb{1}\right)-p(\mathbb{1} \otimes \mathbb{1})+p\left(\Pi_{1} \otimes \Pi_{1}\right)+p\left(\Pi_{0} \otimes \Pi_{0}\right)\right] \\
& =\frac{t}{n}\left(\begin{array}{cccc}
0 & i k & -i k & 0 \\
i k & -p & 0 & -i k \\
-i k & 0 & -p & i k \\
0 & -i k & i k & 0
\end{array}\right) .
\end{aligned}
$$

Note that for $p=0$, we have $\left[e^{\mathscr{I}}\right]^{\otimes n}=\left[e^{-i t \sigma_{x}} \otimes e^{i t \sigma_{x}}\right]^{\otimes n}$, which is exactly the superoperator formulation of the dynamics of the non-decohering walk. So far we have shown that the walk with decoherence is still equivalent to $n$ non-interacting single-qubit systems. We now analyse the behaviour of a single-qubit system under the superoperator $e^{\mathscr{f}}$. The structure of this single particle walk will then allow us to draw immediate conclusions about the entire system.

The eigenvalues of $\mathscr{J}$ are $0,-\frac{p t}{n}, \frac{-p t-i \beta t}{2 n}$ and $\frac{-p t+i \beta t}{2 n}$. Here $\beta=\sqrt{16 k^{2}-p^{2}}$ is a complex constant that will later turn out to be important in determining the behaviour of the system as a function of the rate of decoherence $p$ and the energy $k$. The matrix exponential of $\mathscr{J}$ in this spectral basis can be computed by inspection. To see how our superoperator acts on a density matrix $\boldsymbol{\rho}_{0}$, we may change $\boldsymbol{\rho}_{0}$ to the spectral basis, apply the diagonal superoperator to yield $\rho(t)$, and finally change $\rho(t)$ back to the computational basis. At that point we can apply the usual projectors $\Pi_{0}$ and $\Pi_{1}$ to determine the probabilities of measuring 0 or 1 as a function of time. The result (see Alagic and Russell (2005) for details) is

$$
\begin{aligned}
& P[0]=\frac{1}{2}+\frac{1}{2} e^{-p t / 2 n}\left[\cos \left(\frac{\beta t}{2 n}\right)+\frac{p}{\beta} \sin \left(\frac{\beta t}{2 n}\right)\right] \\
& P[1]=\frac{1}{2}-\frac{1}{2} e^{-p t / 2 n}\left[\cos \left(\frac{\beta t}{2 n}\right)+\frac{p}{\beta} \sin \left(\frac{\beta t}{2 n}\right)\right] .
\end{aligned}
$$

All the properties of the decohering walk can be deduced from these equations. Just as we observed in the mixing behaviour of cycles, the decoherence exhibits three distinctive regimes analogous to underdamping, critical damping and overdamping in a damped oscillator. We will now describe the properties of these regimes.

The underdamping regime occurs for $p<4 k$. To determine the mixing times, we solve $P[0]=P[1]=\frac{1}{2}$, giving exact instantaneous mixing times at

$$
M_{\mathrm{inst}}=\frac{n\left(2 \pi c-\arccos \left(p^{2} / 8 k^{2}-1\right)\right)}{\sqrt{16 k^{2}-p^{2}}}
$$

for all $c \in \mathbb{Z}, c>0$. At these times, the total variational distance between the walk distribution and the uniform distribution is zero, but the walk 'unmixes' again as time progresses. This corresponds to the result in Moore and Russell (2002) for pure state quantum walks, and extends it to regions with small decoherence rates. These mixing times scaling linearly with $n$ represent a quantum improvement over the classical random walk mixing time of $\Theta(n \log n)$, with the caveat that the classical mixing time is not instantaneous, so, strictly speaking, we are not comparing like with like, since in the 
quantum case one must sample at exactly the right moment in time. Note that these periodic mixing times decay with $p$ and disappear altogether when $p \geqslant 4 k$, so the quantum advantage tails off before the critical damping point is reached. Richter (2007b) offers a weak bound of $O\left(n^{3 / 2} \log (1 / \epsilon)\right)$ for the mixing time at around the critical damping point, which is slower than classical.

By examining the local maxima of $P[1]$, we can determine that the walk has approximate instantaneous hitting times to the opposite corner $(1, \ldots, 1)$ at times

$$
H_{\text {one-shot }}(r)=\frac{2 \pi n(2 c+1)}{\sqrt{16 k^{2}-p^{2}}}
$$

for all $c \in \mathbb{Z}, c \geqslant 0$. However, the probability $r(p, c)$ of measuring an exact hit decays exponentially:

$$
r(p, c)=\left[\frac{1}{2}+\frac{1}{2} \exp \left(-p \pi(2 c+1) / \sqrt{16 k^{2}-p^{2}}\right)\right]^{n} .
$$

When no decoherence is present, the walk hits at

$$
H_{\text {one-shot }}(1)=\frac{n \pi(2 c+1)}{2 k},
$$

and it does so exactly, that is, $r(c)=1$. This corresponds to the result for the discrete-time quantum walk given in Kempe (2003b; 2005), see Section 5.6, so both discrete-time and continuous-time quantum walks on the hypercube exhibit exponentially faster hitting times than classical random walks. The difference is that the continuous-time quantum walk hits exactly, while the discrete-time quantum walk hits with a probability that is less than one: see Kempe (2003b; 2005) for details.

For a fixed $p \geqslant 4 k$, Alagic and Russell (2005) shows that the walk behaves much like the classical walk on the hypercube, the measurement distribution of the walk converges to the uniform distribution in time $M(\epsilon)=\Theta(n \log n)$, just as in the classical case.

As $p \rightarrow \infty$, the walk suffers from the quantum Zeno effect. Informally, we can say that the rate of decoherence is so large that the walk is continuously being reset to the initial wave function $|0\rangle^{\otimes n}$ by measurement. By inspection of equations (119), it is clear that $P[0] \rightarrow 1$ and $P[1] \rightarrow 0$ as $p \rightarrow \infty$.

This concludes our overview of decoherence in continuous-time walks. We have observed some of the same effects of enhanced mixing and smoother spreading, and also shown that where the decoherence does not directly help, the quantum speed up is at least robust under small amounts of decoherence.

\section{Quantum walks in physical systems}

As well as the potential for algorithmic applications that has been in the background of most of our discussion of decoherence in quantum walks in this review, physical systems can be made to perform a quantum walk, and this process is of interest in its own right as an example of precise coherent control. Indeed, the first suggested application of a quantum walk in a physical system was to test decoherence rates in a single trapped ion. Travaglione and Milburn (2002) described how a coined quantum walk can be 
performed using the vibration modes of the trapped ion coupled to an internal state of the ion as the quantum coin. They note that a cycle of size $N=4$ performed in this way should exhibit periodic behaviour, see Section 2.3. Sanders et al. (2003) suggested a quantum walk using the phase of the electromagnetic field in a cavity as the position (so the walk is again on a cycle) with the role of the coin played by an atom, also in the cavity, which couples to the cavity field depending on its internal state. Since the cavity field cannot be in a state localised at a point in phase space, the positions must be approximated by small regions that do not overlap. Sanders et al. provided a detailed analysis of the experimental parameters necessary to maintain distinct positions during the quantum walk. Dür et al. (2002) proposed a quantum walk on the line using an atom hopping between traps in an optical lattice. The role of the coin is again played by the internal state of the atom. One of the potential sources of error in this system is imperfect transitions between lattice sites, so, along with analysing errors due to imperfect Hadamard operations, Dür et al. also considered the effects of imperfect shift operations. Their work is numerical, but shows that imperfect shifts, or spontaneous tunnelling, smear out the distribution without necessarily reducing the quantum features. This analysis is typical of the extra factors that need to be considered in experimental systems. Since the quantum walk takes place in a subspace of the full quantum system, many of the potential sources of decoherence take the system outside of the subspace of the quantum walk, so the resulting decohered dynamics is not a classical random walk. All of these proposals are within current experimental capabilities but, to date, none has been carried out.

The examples given so far have all been discrete-time coined quantum walks. Solenov and Fedichkin $(2006 \mathrm{a}$; 2006b) analysed a ring of quantum dots in which the electrons in the dots perform a continuous-time quantum walk. They applied perturbation theory techniques similar to those described in Section 6.2 to analyse the effects of decoherence.

There are a number of ways in which photons can be made to follow a quantum walk dynamics, and while some have been implemented, all have used high light intensities that admit a classical wave description. The earliest example, which predates the algorithmic discussions of quantum walks, is in Bouwmeester et al. (1999), where the twin peaked distribution of a quantum walk on the line was presented but without identifying it as such. Knight et al. (2003) discusses this experiment, arguing that quantum walks on the line are actually not quantum at all. Kendon and Sanders (2004) explained how what Bouwmeester et al. did can be regarded as a quantum walk and suggested an enhanced experiment, in which monitoring the individual photon 'walkers' reduces the dynamics to a classical random walk, to distinguish a classical 'wave walk' from a quantum walk.

A quantum walk experiment carried out by Ryan et al. used a 3 qubit NMR system to perform a quantum walk on a cycle of size $N=4$ (Ryan et al. 2005). This is actually a quantum computation of a quantum walk, since the three qubits are used to represent the binary number labelling the vertex (two qubits), and the qubit coin. This is distinctly different from the physical systems described above, see Kendon (2006b) for more discussion on this point. Ryan et al. also applied decoherence artificially to the qubits to observe that the quantum walk degrades into the classical walk. 


\section{Quantum walks on directed graphs}

Providing a quantum walk dynamics on undirected graphs is straightforward since the adjacency matrix of an undirected graph is symmetric, hence it can be turned into a unitary (with added coin) or Hermitian operator for any such graph. In contrast, determining whether a directed graph allows a unitary dynamics that respects the graph structure is a hard problem, to which a general answer is not known. Aharonov et al. (2001) gave a useful definition of how a quantum walk dynamics should respect the structure of a graph, though other approaches are possible, see, for example, Montanaro (2007). Severini (2003; 2006) provide some necessary conditions, by studying the pattern of a unitary matrix, defined as $U_{i j}^{(P)}=1$ if $U_{i j} \neq 0$ and $U_{i j}^{(P)}=0$ if $U_{i j}=0$. If $A=U^{(P)}$ for some $U$, a quantum walk can naturally be applied to the graph with adjacency matrix $A$. The situation is simpler for some restricted types of graphs, such as line graphs. A line graph $L^{(G)}$ is obtained from graph $G$ by making each edge in $G$ a vertex in $L^{(G)}$, and edges for each pair of edges in $G$ that are adjacent. If $x, y, z$ are vertices in $G$ connected by directed edges $(x y)$ and $(y z)$ that can be traversed only from $x$ to $y$ to $z$, then the vertices in $L^{(G)}$ correspond to all $(x y) \in G$, and the edges in $L^{(G)}$ correspond to all $((x y)(y z))$ where $(x y)$ and $(y z)$ are edges in $G$. This definition can be applied recursively to generate a family of line graphs from a single initial graph $G$, and Pakoński et al. (2003) provides conditions under which line graphs can be quantised.

Starting with an undirected graph, a corresponding directed graph can be constructed by a mapping due to Watrous (2001), which is similar to the line graph definition above, except that each undirected edge is treated as a pair of directed edges. The resulting directed graph is always the pattern of a unitary matrix. The corresponding dynamics, using an associated unitary matrix as the step operator, corresponds exactly to the coined quantum walk dynamics (with a Grover coin) on the original undirected graph (Watrous 2002).

Directed graphs that do not have the pattern of a unitary matrix may still admit a quantum walk dynamics. Montanaro (2007) defines 'reversibility' for a directed graph, and proves this allows for a unitary quantum walk in which the coin selects between different sets of cycles in the graph, rather than different paths from each vertex. For irreversible graphs, Montanaro suggests splitting the graph into subgraphs that are reversible, on which the quantum walk dynamics can be unitary, combined with a non-unitary step (a partial measurement) to switch between subgraphs in an irreversible way that, nonetheless, preserves coherence within each reversible subgraph. If one is willing to allow some transitions that are between vertices not connected by edges, the method of Szegedy (2004a) also provides a quantum Markov process on directed graphs.

This brief section has only touched on the range of problems in the realm of directed graphs, reflecting the limited work done in this area. For example, no study of decoherence in quantum walks on directed graphs has been done, nor consideration of the class of graphs that might be the pattern of a CP map (rather than of a unitary matrix). 


\section{Summary and outlook}

We have seen in our broad-ranging review of decoherence in quantum walks that the effects are both non-trivial and have potential algorithmic applications. As the decoherence rate is increased, for moderate timescales the quantum features persist, usually to a point of 'critical damping', after which the behaviour resembles that of a classical system. In the quantum region before the critical damping point, the useful algorithmic properties, such as spreading and mixing, are often better than the pure quantum dynamics. Indeed, for cycles and tori, some element of non-unitary evolution is essential for efficient mixing behaviour. Our tentative conclusion is thus that decoherence is a useful enhancement, and non-unitary dynamics are a promising avenue of enquiry in the quest for practical applications of quantum computing. The incomplete nature of our conclusions is in part a reflection of our limited knowledge of the conditions under which a quantum speed up can be obtained.

The idea that the addition of classical randomness can enhance a useful algorithmic property also occurs in other settings, both quantum and classical. An example from the field of quantum information may be found in Kraus et al. (2005) and Renner et al. (2005), where the security of a quantum secret key is increased by adding a small amount of randomness to the key during the privacy amplification stage of the protocol. This can be understood in two ways. The goal is to reduce the amount of information available to an eavesdropper to a trivial level. The added randomness reduces the final size of the secret key, but it reduces even more the information that the eavesdropper has. This works for both classical and quantum keys. From a quantum perspective, the randomness takes the form of entanglement with an environment that the eavesdropper does not have access to. Since the total amount of entanglement is limited, this reduces the entanglement of the key with the eavesdropper, and thus reduces the information available to the eavesdropper. The interpretation of this in the context of quantum walks is of course rather different, but the common theme is that the decoherence or randomness induces a more uniform distribution, in this case in the form of a smoother 'top-hat' distribution in the walk on the line, and faster mixing times on finite graphs. Some further insight is provided in Kendon and Maloyer (2006), where the entanglement between the coin and the position is used to gauge the impact of the added randomness. The optimal decoherence rate turns out just to remove all the quantum entanglement, and can be thought of as removing the 'extra' quantum correlations that would otherwise keep the distribution away from uniform.

As will have become clear by now, there are many gaps in the analysis of decoherence in quantum walks, and, more importantly, many gaps in our understanding of what might make a useful quantum walk algorithm. Some of these shortcomings will only be accessible to serious study when (if) we have a larger (that is, larger than we can simulate classically) quantum computer available to test the performance of actual algorithms. In the meantime, there is still much useful progress that can be made, in particular, in understanding the role of symmetry in the properties of quantum walks. Many of the examples studied so far turn out on closer inspection to be exceptional cases, in that a slight change in the symmetry of the dynamics or initial state can reverse the 
interesting quantum properties. The role of decoherence as a 'memory eraser' to eliminate the dependence on the initial state, while still retaining the interesting quantum properties, is thus one of its most appealing features. My hope in offering this review is that it will be a useful summary of the current state of research in a field that has seen significant recent activity and advances, and that it will facilitate and spur further efforts in this area.

\section{Acknowledgements}

I gratefully thank my colleagues for many useful and stimulating discussions of quantum walks, especially Dorit Aharonov, Andris Ambainis, Todd Brun, Ivens Carneiro ${ }^{\dagger}$, Hilary Carteret, Andrew Childs, Richard Cleve, Jochen Endrejat, Ed Farhi, Will Flanagan, Mathieu Girerd, Mark Hillery, Peter Høyer, Julia Kempe, Peter Knight, Norio Konno, Barbara Kraus, Meng Loo, Rik Maile, Olivier Maloyer, Cris Moore, Peter Richter, Eugenio Roldán, Alex Russell, Barry Sanders, Mario Szegedy, John Sipe, Tino Tamon, Ben Tregenna, John Watrous, and Xibai Xu.

\section{References}

Adamczak, W., Andrew, K., Hernberg, P. and Tamon, C. (2003) A note on graphs resistant to quantum uniform mixing. ArXiv: quant-ph/0308073.

Adamczak, W., Andrew, K., Bergen, L., Ethier, D., Hernberg, P., Lin, J. and Tamon, C. (2007) Non-uniform mixing of quantum walk on cycles. Intl. J. Quantum Inf. (to appear). See also ArXiv: 0708.2096.

Aharonov, D., Ambainis, A., Kempe, J. and Vazirani, U. (2001) Quantum walks on graphs. In: Proc. 33rd Annual ACM STOC., ACM 50-59.

Aharonov, Y., Davidovich, L. and Zagury, N. (1992) Quantum random walks. Phys. Rev. A 48 (2) 1687-1690.

Ahmadi, A., Belk, R., Tamon, C. and Wendler, C. (2003) On mixing in continuous-time quantum walks on some circulant graphs. Quantum Information and Computation 3 (6) 611-618.

Alagic, G. and Russell, A. (2005) Decoherence in quantum walks on the hypercube. Phys. Rev. A 720062304.

Ambainis, A. (2003) Quantum walks and their algorithmic applications. Intl. J. Quantum Information 1 (4) 507-518.

Ambainis, A. (2004) Quantum walk algorithms for element distinctness. In: 45th Annual IEEE Symposium on Foundations of Computer Science, IEEE Computer Society Press 22-31.

Ambainis, A., Bach, E., Nayak, A., Vishwanath, A. and Watrous, J. (2001) One-dimensional quantum walks. In: Proc. 33rd Annual ACM STOC., ACM 60-69.

Anderson, P. W. (1958) Absence of diffusion in certain random lattices. Phys. Rev. 109 (5) 1492-1505.

Bach, E., Coppersmith, S., Goldschen, M.P., Joynt, R. and Watrous, J. (2004) One-dimensional quantum walks with absorbing boundaries. J. Comput. Syst. Sci. 69 (4) 562-592.

Bednarska, M., Grudka, A., Kurzyński, P., Łuczak, T. and Wójcik, A. (2003) Quantum walks on cycles. Phys. Lett. A 317 (1-2) 21-25.

Bennett, C.H., Bernstein, E., Brassard, G. and Vazirani, U. (1997) Strengths and weaknesses of quantum computing. SIAM J. Comput. 26 (5) 151-152.

$\dagger$ It was with much sadness that we learned of the death of Ivens Carneiro in a traffic accident in April 2006. 
Bouwmeester, D., Marzoli, I., Karman, G. P., Schleich, W. and Woerdman, J. P. (1999) Optical Galton board. Phys. Rev. A 61013410.

Brun, T.A., Carteret, H.A. and Ambainis, A. (2003a) Quantum random walks with decoherent coins. Phys. Rev. A 67032304.

Brun, T. A., Carteret, H. A. and Ambainis, A. (2003b) The quantum to classical transition for random walks. Phys. Rev. Lett. 91 (13) 130602.

Brun, T. A., Carteret, H. A. and Ambainis, A. (2003c) Quantum walks driven by many coins. Phys. Rev. A 67052317.

Carlson, W., Ford, A., Harris, E., Rosen, J., Tamon, C. and Wrobel, K. (2006) Universal mixing of quantum walk on graphs. ArXiv: quant-ph/0608044.

Carneiro, I., Loo, M., Xu, X., Girerd, M., Kendon, V. M. and Knight, P. L. (2005) Entanglement in coined quantum walks on regular graphs. New J. Phys. 756.

Carteret, H. A., Ismail, M. A. and Richmond, B. (2003) Three routes to the exact asymptotics for the one-dimensional quantum walk. J. Phys. A 36 (33) 8775-8795.

Childs, A. and Eisenberg, J. M. (2005) Quantum algorithms for subset finding. Quantum Information and Computation 5 593-604.

Childs, A. and Goldstone, J. (2004a) Spatial search by quantum walk. Phys. Rev. A 70022314.

Childs, A. M. and Goldstone, J. (2004b) Spatial search and the Dirac equation. Phys. Rev. A 70 042312.

Childs, A. M., Cleve, R., Deotto, E., Farhi, E., Gutmann, S. and Spielman, D. A., (2003) Exponential algorithmic speedup by a quantum walk. In: Proc. 35th Annual ACM STOC., ACM 59-68.

Dür, W., Raussendorf, R., Kendon, V.M. and Briegel, H.-J. (2002) Quantum random walks in optical lattices. Phys. Rev. A 66052319.

Dyer, M., Frieze, A. and Kannan, R. (1991) A random polynomial-time algorithm for approximating the volume of convex bodies. J. of the ACM 38 (1) 1-17.

Ermann, L., Paz, J.P. and Sraceno, M. (2006) Decoherence induced by a chaotic environment: a quantum walker with a complex coin. Phys. Rev. A 73 (1) 012302.

Farhi, E. and Gutmann, S. (1998) Quantum computation and decison trees. Phys. Rev. A 58 915-928.

Fedichkin, L., Solenov, D. and Tamon, C. (2006) Mixing and decoherence in continuous time quantum walks on cycles. Quantum Information and Computation 6 (3) 263-276.

Feldman, E. and Hillery, M. (2004) Scattering theory and discrete-time quantum walks. Phys. Lett. A 324 (3) 277.

Feynman, R. P. (1986) Quantum mechanical computers. Found. Phys. 16507.

Feynman, R. P., Leighton, R. B. and Sands, M. (1964) Feynman Lectures on Physics, Addison Wesley.

Flitney, A. P., Abott, D. and Johnson, N. F. (2004) Quantum random walks with history dependence. J. Phys. A 37 7581-7591.

Gottlieb, A. D. (2004) Two examples of discrete-time quantum walks taking continuous steps. Phys. Rev. E 72 (4) 047102.

Gottlieb, A. D., Janson, S. and Scudo, P.F. (2005) Convergence of coined quantum walks in $\mathbb{R}^{d}$. Inf. Dimen. Anal. Quantum Probab. Rel. Topics 8 (1) 129-140.

Grimmett, G., Janson, S. and Scudo, P. (2004) Weak limits for quantum random walks. Phys. Rev. E 69026119.

Grossing, G. and Zeilinger, A. (1988) Quantum cellular automata. Complex Systems 2 197-208.

Grover, L.K. (1996) A fast quantum mechanical algorithm for database search. In: Proc. 28th Annual ACM STOC., ACM 212.

Gudder, S. (1988) Quantum Probability, Academic Press.

Gurvitz, S. A. (1997) Measurements with a noninvasive detector and dephasing mechanism. Phys. Rev. B 5615215. 
Gurvitz, S. A., Fedichkin, L., Mozyrsky, D. and Berman, G.P. (2003) Relaxation and Zeno effects in qubit measurements. Phys. Rev. Lett. 91066801.

Inui, N., Konishi, Y. and Konno, N. (2004) Localization of two-dimensional quantum walks. Phys. Rev. A 69052323.

Jerrum, M., Sinclair, A. and Vigoda, E. (2001) A polynomial-time approximation algorithm for the permanent of a matrix with non-negative entries. In: Proc. 33rd Annual ACM STOC., ACM $712-721$.

Keating, J. P., Linden, N., Matthews, J. C. F. and Winter, A. (2006) Localization and its consequences for quantum walk algorithms and quantum communication. ArXiv: quant-ph/0606205.

Kempe, J. (2003a) Quantum random walk algorithms. Contemp. Phys. 44 (3) 302-327.

Kempe, J. (2003b) Quantum random walks hit exponentially faster. In: Proc. 7th Intl. Workshop on Randomization and Approximation Techniques in Computer Science (RANDOM '03). SpringerVerlag Lecture Notes in Computer Science 354-369.

Kempe, J. (2005) Quantum random walks hit exponentially faster. Probability Th. and Related Fields 133 (2) 215-235.

Kendon, V. (2006a) Quantum walks on general graphs. Int. J. Quantum Inf. 4 (5) 791-805. See also ArXiv: quant-ph/0306140.

Kendon, V. (2006b) A random walk approach to quantum algorithms. Phil. Trans. Roy. Soc. A 364 3407-3422.

Kendon, V. and Maloyer, O. (2006) Optimal computation with non-unitary quantum walks. ArXiv: quant-ph/0610240. To appear in Theor. Comp. Sci. A (2008) as a postproceedings volume for CiE 2006.

Kendon, V. and Tregenna, B. (2002) Decoherence in a quantum walk on a line. In: Shapiro, J.H. and Hirota, O. (eds.) Quantum Communication, Measurement and Computing (QCMC'02), Rinton Press 463.

Kendon, V. and Tregenna, B. (2003) Decoherence can be useful in quantum walks. Phys. Rev. A 67 042315.

Kendon, V. M. and Sanders, B.C. (2004) Complementarity and quantum walks. Phys. Rev. A 71 022307.

Knight, P.L., Roldán, E. and Sipe, J.E. (2003) Quantum walk on the line as an interference phenomenon. Phys. Rev. A 68 020301(R).

Knight, P. L., Roldán, E. and Sipe, J.E. (2004) Propagating quantum walks: the origin of interference structures. J. Mod. Opt. 51 1761-1777.

Konno, N. (2002) Quantum random walks in one dimension. Quantum Information Processing 1 (5) $345-354$.

Konno, N. (2005a) A new type of limit theorems for the one-dimensional quantum random walk. Journal of the Mathematical Society of Japan 57 (4) 1179-1195.

Konno, N. (2005b) A path integral approach for disordered quantum walks in one dimension. Fluctuation and Noise Letters 5 (4) 529-537.

Konno, N., Namiki, T. and Soshi, T. (2004) Symmetricity of distribution for the one-dimentional Hadamard walk. Interdisciplinary Infor. Sci. 10 (1) 11-22.

Kottos, T. and Smilansky, U. (1997) Quantum chaos on graphs. Phys. Rev. Lett. 79 4794-4797.

Košík, J., Bužek, V. and Hillery, M. (2006) Quantum walks with random phase shifts. Phys. Rev. A 74 (2) 022310.

Kraus, B., Gisin, N. and Renner, R. (2005) Lower and upper bounds on the secret key rate for QKD protocols using one-way classical communication. Phys. Rev. Lett. 95080501.

Krovi, H. and Brun, T. A. (2006a) Hitting time for quantum walks on the hypercube. Phys. Rev. A 73 (3) 032341. 
Krovi, H. and Brun, T. A. (2006b) Quantum walks with infinite hitting times. Phys. Rev. A 74 (4) 042334.

Lo, P., Rajaram, S., Schepens, D., Sullivan, D., Tamon, C. and Ward, J. (2006) Mixing of quantum walk on circulant bunkbeds. Quantum Information and Computation 6 (4-5) 370-381.

Lomont, C. (2004) The hidden subgroup problem - review and open problems. ArXiv: quant-ph/0411037.

Lopéz, C. C. and Paz, J.P. (2003) Decoherence in quantum walks: Existence of a quantum-classical transition. Phys. Rev. A $\mathbf{6 8} 052305$.

Mackay, T. D., Bartlett, S. D., Stephenson, L. T. and Sanders, B. C. (2002) Quantum walks in higher dimensions. J. Phys. A: Math. Gen. 352745.

Magniez, F., Santha, M. and Szegedy, M. (2005) Quantum algorithms for the triangle problem. In: Proceedings of 16th ACM-SIAM Symposium on Discrete Algorithms, Society for Industrial and Applied Mathematics, Philadelphia 1109-1117.

Maloyer, O. and Kendon, V. (2007) Decoherence vs entanglement in coined quantum walks. New J. Phys. 987.

Meyer, D. A. (1996a) From quantum cellular automata to quantum lattice gases. J. Stat. Phys. 85 551-574.

Meyer, D. A. (1996b) On the absence of homogeneous scalar unitary cellular automata. Phys. Lett. A 223 (5) 337-340.

Misra, B. and Sudarshan, E. C. G. (1977) The Zeno's paradox in quantum theory. J. Math. Phys. 18 756.

Montanaro, A. (2007) Quantum walks on directed graphs. Quantum Information and Computation 7 (1-2) 93-102.

Moore, C. and Russell, A. (2002) Quantum walks on the hypercube. In: Rolim, J. D. P. and Vadhan, S. (eds.) Proc. 6th Intl. Workshop on Randomization and Approximation Techniques in Computer Science (RANDOM '02), Springer 164-178.

Motwani, R. and Raghavan, P. (1995) Randomized Algorithms, Cambridge University Press.

Nayak, A. and Vishwanath, A. (2000) Quantum walk on the line. ArXiv: quant-ph/0010117.

Pakoński, P., Tanner, G. and Życzkowski, K. (2003) Families of line-graphs and their quantization. J. Stat. Phys 111 (5/6) 1331-1352.

Renner, R., Gisin, N. and Kraus, B. (2005) An information-theoretic security proof for QKD protocols. Phys. Rev. A 72012332.

Ribeiro, P., Milman, P. and Mosseri, R. (2004) Aperiodic quantum random walks. Phys. Rev. Lett. 93 (19) 190503.

Richter, P. (2007a) Almost uniform sampling in quantum walks. New J. Phys. 9 72. See also ArXiv: quant-ph/0606202.

Richter, P. (2007b) Quantum speedup of classical mixing processes. Phys. Rev. A 76042306 . See also ArXiv: quant-ph/0609204.

Romanelli, A., Sicardi-Schifino, A.C., Siri, R., Abal, G., Auyuanet, A. and Donangelo, R. (2004) Quantum random walk on the line as a Markovian process. Physica A 338 395-405.

Romanelli, A., Siri, R., Abal, G., Auyuanet, A. and Donangelo, R. (2003) Decoherence in the quantum walk on the line. Physica A 347 137-152.

Ryan, C. A., Laforest, M., Boileau, J.C. and Laflamme, R. (2005) Experimental implementation of discrete time quantum random walk on an NMR quantum information processor. Phys. Rev. A 72062317.

Sachdev, S. (1999) Quantum Phase Transitions, Cambridge University Press.

Sanders, B.C., Bartlett, S. D., Tregenna, B. and Knight, P. L. (2003) Quantum quincunx in cavity QED. Phys. Rev. A 67042305. 
Schöning, U. (1999) A probabilistic algorithm for $k$-SAT and constraint satisfaction problems. In: 40th Annual Symposium on FOCS, IEEE Computer Society Press 17-19.

Severini, S. (2003) On the digraph of a unitary matrix. SIAM J. Matrix Anal. Appl. 25 (1) 295-300.

Severini, S. (2006) Graphs of a unitary matrix. ArXiv: math.C0/0303084.

Shapira, D., Biham, O., Bracken, A. J. and Hackett, M. (2003) One dimensional quantum walk with unitary noise. Phys. Rev. A 68 (6) 062315.

Shenvi, N., Kempe, J. and Birgitta Whaley, K. (2003) A quantum random walk search algorithm. Phys. Rev. A 67052307.

Shor, P. W. (1997) Polynomial-time algorithms for prime factorization and discrete logarithms on a quantum computer. SIAM J. Sci Statist. Comput. 261484.

Solenov, D. and Fedichkin, L. (2006a) Continuous-time quantum walks on a cycle graph. Phys. Rev. A 73012313 .

Solenov, D. and Fedichkin, L. (2006b) Non-unitary quantum walks on hyper-cycles. Phys. Rev. A 73012308.

Strauch, F. W. (2006a) Connecting the discrete and continuous-time quantum walks. Phys. Rev. A 74 (3) 030301.

Strauch, F. W. (2006b) Relativistic quantum walks. Phys. Rev. A 73 (6) 054302.

Szegedy, M. (2004a) Quantum speed-up of Markov chain based algorithms. In: 45th Annual IEEE Symposium on Foundations of Computer Science, IEEE Computer Society Press 32-41.

Szegedy, M. (2004b) Spectra of quantized walks and a $\sqrt{\delta \epsilon}$ rule. ArXiv: quant-ph/0401053.

Tadej, W. and Życzkowski, K. (2006) A concise guide to complex Hadamard matrices. Open Syst. Inf. Dyn. 13 133-177.

Travaglione, B. C. and Milburn, G. J. (2002) Implementing the quantum random walk. Phys. Rev. A 65032310 .

Tregenna, B., Flanagan, W., Maile, R. and Kendon, V. (2003) Controlling discrete quantum walks: coins and initial states. New J. Phys. 583.

Watrous, J. (2001) Quantum simulations of classical random walks and undirected graph connectivity. J. Comp. System Sciences 62 (2) 376-391.

Watrous, J. (2002) Private communication.

Weiss, G. H. (1994) Aspects and Applications of the Random Walk, North-Holland.

Yamasaki, T., Kobayashi, H. and Imai, H. (2002) An analysis of absorbing times of quantum walks. In: Calude, C., Dinneen, M. J. and Peper, F. (eds.) Proceedings: Unconventional Models of Computation, Third Intl. Conf., UMC 2002. Springer-Verlag Lecture Notes in Computer Science $2509315-330$. 